\title{
NUMERICAL RECONSTRUCTION BASED ON CARLEMAN ESTIMATES OF A SOURCE TERM IN A REACTION-DIFFUSION EQUATION*
}

\author{
Muriel Boulakia ${ }^{1, * *}$, Maya de Buhan ${ }^{2}$ and Erica L. Schwindt ${ }^{3}$
}

\begin{abstract}
In this article, we consider a reaction-diffusion equation where the reaction term is given by a cubic function and we are interested in the numerical reconstruction of the time-independent part of the source term from measurements of the solution. For this identification problem, we present an iterative algorithm based on Carleman estimates which consists of minimizing at each iteration cost functionals which are strongly convex on bounded sets. Despite the nonlinear nature of the problem, we prove that our method globally converges and the convergence speed evaluated in weighted norm is linear. In the last part of the paper, we illustrate the effectiveness of our method with several numerical reconstructions in dimension one or two.
\end{abstract}

Mathematics Subject Classification. 35R30, 35K55, 35K57, 93B07.

Received July 17, 2019. Accepted November 30, 2020.

\section{INTRODUCTION}

Let $\Omega$ be a $C^{2}$ bounded domain of $\mathbb{R}^{d}$ for $d=1,2$ or 3 and $T>0$. We consider the following reaction-diffusion equation

$$
\begin{cases}\partial_{t} u(t, x)-\Delta u(t, x)+u^{3}(t, x)=\sigma(x) h(t, x), & (t, x) \in(0, T) \times \Omega, \\ u(t, x)=g(t, x), & (t, x) \in(0, T) \times \partial \Omega, \\ u(0, x)=u_{\circ}(x), & x \in \Omega,\end{cases}
$$

where $g$ is the Dirichlet boundary data and $u_{\circ}$ is the initial condition. In the right hand side of the first equation, we assume that the time-dependent function $h$ is known and we focus on the reconstruction of $\sigma$ which is assumed to depend only on the spatial variable. To identify this unknown, we have two kinds of measurements, the flux

* This work was partially funded by the DGA 2014-91-00-79 project. The second author was partially supported by the Project "Analysis and simulation of optimal shapes - application to life sciences" of the Paris City Hall.

Keywords and phrases: Inverse problems, nonlinear parabolic equations, Carleman estimates, numerical reconstruction.

1 Sorbonne Université, CNRS, Université de Paris, Inria, Laboratoire Jacques-Louis Lions, équipe COMMEDIA, 75005 Paris, France.

2 Université Paris-Saclay, CNRS, Laboratoire de mathématiques d'Orsay, 91405, Orsay, France.

3 Inria Nancy Grand-Est and CNRS UMR 7503 LORIA, Villers-lès-Nancy, France.

** Corresponding author: boulakia@ljll.math.upmc.fr 
of the solution on a part of a boundary and the solution in the whole domain at a given time:

$$
\begin{cases}m(t, x):=\nabla u(t, x) \cdot n(x), & (t, x) \in(0, T) \times \Gamma, \\ r(x):=u\left(T_{0}, x\right), & x \in \Omega,\end{cases}
$$

where $\Gamma \subset \partial \Omega, T_{0} \in(0, T)$ and $n$ is the outward-pointing unit normal vector defined on $\partial \Omega$.

Regarding the applications, this model can represent the evolution of a pollutant in the atmosphere. The source in the right hand side corresponds to a spill of pollutant and we want to localise it. This model can also be viewed as a simplified model to represent the evolution of the electrical potential in the heart (we refer to [8] for a detailed presentation of this application domain and more precisely to Section 2.9.7 of [8] for cubic-like reaction models). In our model, the natural propagation of the potential is initiated by the initial condition and the source in the right hand side may correspond to a secondary undesirable source that we want to identify.

Let $\sigma_{\max }>0$ be a fixed constant. We assume that the source term $\sigma$ that we want to reconstruct belongs to $L^{\infty}(\Omega)$ and satisfies the following a priori bound:

$$
\|\sigma\|_{L^{\infty}(\Omega)} \leq \sigma_{\max }
$$

For this problem, according to Bukhgeim-Klibanov method, $\sigma$ is uniquely determined by the measurements and a Lipschitz stability estimate holds under appropriate assumptions on the data (the precise result is stated in Prop. 2.6). Bukhgeim-Klibanov method [5] is a classical theoretical method to prove the uniqueness and stability for parameter identification problems. For a presentation of this method which relies on Carleman estimates and for a survey on its applications, we refer to [18] and in particular to Section 3.3 on parabolic equations. For the inverse problem of coefficients identification in nonlinear parabolic equations, let us in particular mention that $[3,9]$ deal with the theoretical stability of the reaction term in a semi-linear PDE.

In this paper, our aim is to tackle the numerical reconstruction of $\sigma$ and to propose for this nonlinear problem a globally convergent method. Our work is drawn from a numerical method presented in [1] for the identification of a potential in a wave equation. The method strongly relies on Carleman inequalities and it consists of an iterative algorithm minimizing at each iteration a cost functional involving Carleman weights. The main strength of this numerical method is that it globally converges to the exact solution i.e. it converges independently of the initialization. In particular, contrary to classical minimization techniques like Tikhonov methods [16], it is not necessary to add a priori knowledge on the source term through the data of a background state to convexify the cost functional.

As pointed out in the introduction of [2], this method induces several numerical challenges. In particular, the classical Carleman weights have very strong variations due to the presence of a double exponential involving large coefficients. That is why, as in [2] for the wave equations, we need to construct new Carleman weights for the heat equation which involve single exponentials (these weights are given by (2.3) and (2.4)).

The presence of a nonlinearity in our PDE leads to additional difficulties in the study of the numerical methods. In particular, the strong convexity properties of the cost functional are restricted to bounded spaces. Moreover, the operator appearing in the cost functional has to be modified by adding truncation operators in the nonlinear terms to tackle these terms in the proof of the convergence of the numerical method. At last, contrary to [2] where the PDE is linear, introducing a conjugate variable $e^{s \varphi} z$ does not allow to overcome the fact that, even with single exponentials, the minimization of the cost functional is challenging.

Let us mention that we could have considered general cubic functions of the form

$$
a_{3} u^{3}+a_{2} u^{2}+a_{1} u
$$

with $a_{3}>0$ instead of a simple cubic monomial in this semi-linear parabolic partial differential equations (1.1). By this way, the study includes the bistable equation or Allen-Cahn equation. Moreover, we refer to Remark 2.4 for some remarks on the case of other boundary data (Neumann boundary conditions instead of the second equation in (1.1) and boundary measurements on the solution itself). 
Using Carleman estimates to solve numerically inverse problems has been first considered in the paper [17] by Klibanov. This method called convexification method has been applied in several papers. In [19], the authors are interested by the reconstruction of a coefficient in a parabolic equation and present a gradient method applied to a strictly convex cost functional involving Carleman weights. In [22], the authors consider the reconstruction of the initial condition in a nonlinear parabolic equation. We also refer to [20] for the most recent paper which applies this convexification method.

For numerical studies applying Carleman estimates to controllability problems, we refer to [7] for the numerical controllability of the wave equation and [12] for the numerical controllability of the heat, Stokes and Navier-Stokes equation.

The paper is organized as follows. Sections 2 give some preliminary results. First, in Section 2.1, we present a Carleman estimate for the heat operator with Dirichlet boundary conditions. In this estimate, we consider two kinds of Carleman weights: the classical weights for the heat equation with a double exponential and new weights involving single exponentials which are introduced for numerical purposes. Then, in Section 2.2, we state a regularity result satisfied by the solution of equation (1.1). The proofs of the Carleman estimate and the regularity result are presented in Appendices A and B respectively. At last, in Section 2.3, we state the stability inequality associated to our inverse problem.

Section 3.1 is the core of the paper and presents the numerical reconstruction method of the source term. The latter is an iterative process which requires at each iteration the minimization of a functional based on the Carleman estimate. This section states the global convergence of the method (Theorem 3.4). In Section 3.3, we establish properties satisfied by the functional to minimize at each step. In particular, the existence of a global minimizer of the functional is stated in Lemma 3.7 and the strong convexity on bounded set is proved in Lemma 3.8. In the last section (Sect. 3.4), we prove the global convergence property. Finally, Section 4 is devoted to the implementation of the algorithm and the numerical results obtained for several 1D and $2 \mathrm{D}$ test cases.

\section{Preliminary Results}

\subsection{Carleman inequality for the heat equation}

Without loss of generality, from now on, we assume that $T_{0}=\frac{T}{2}$.

In this section, we state a Carleman inequality for the heat equation in two cases. The first case corresponds to the classical weights with a double exponential while, in the second case, the weights only involve single exponentials as in Section 3 of [27]. Let us specify these two cases:

- Case 1: For $\lambda>0$, we define $\theta$ and $\varphi$ by: for all $(t, x) \in(0, T) \times \Omega$

$$
\theta(t, x)=\frac{e^{\lambda\left(2\left\|\eta_{0}\right\|_{\infty}+\eta_{0}(x)\right)}}{t(T-t)} \quad \varphi(t, x)=\frac{e^{\lambda\left(2\left\|\eta_{0}\right\|_{\infty}+\eta_{0}(x)\right)}-e^{4 \lambda\left\|\eta_{0}\right\|_{\infty}}}{t(T-t)}
$$

where $\eta_{0}$ satisfies the following properties:

$$
\eta_{0}>0 \text { in } \Omega, \quad\left|\nabla \eta_{0}\right| \geq C>0 \text { in } \bar{\Omega} \text { and } \eta_{0}=0 \text { on } \partial \Omega \backslash \Gamma .
$$

- Case 2: For all $(t, x) \in(0, T) \times \Omega$, we define

$$
\theta(t)=\frac{1}{t(T-t)}-\frac{1-\rho}{T_{0}^{2}}
$$

and

$$
\varphi(t, x)=\psi(x) \theta(t) \quad \text { with } \quad \psi(x)=\left|x-x_{0}\right|^{2}-2 \sup _{x \in \bar{\Omega}}\left|x-x_{0}\right|^{2},
$$


where $x_{0}$ is an arbitrary point in $\mathbb{R}^{d} \backslash \bar{\Omega}$ and $\rho$ is a constant satisfying $0<\rho<1$. We notice that $\theta>0$ and $\psi<0$. In this case, we assume in addition that $x_{0}$ and $\Gamma$ are such that

$$
\left\{x \in \partial \Omega \mid\left(x-x_{0}\right) \cdot n(x)>0\right\} \subset \Gamma .
$$

Let us mention that the spatial part $\psi$ of the Carleman weight in Case 2 resembles the one proposed in [1] for the wave equation. Moreover, the geometric condition (2.5) which is classical for the wave equation (see [14, 23]) is unusual for the heat equation and is linked to this new choice of weights. With these weights, we have less flexibility in the computations and we need an extra condition on the measurement domain compared to the classical weights corresponding to Case 1. On the other hand, if we take the classical weights, the presence of a double exponential in the functional to minimize (see (3.11)) is prohibitive to address numerical applications (we refer to Rem. 2.3 for additional comments). In all our numerical tests presented in Section 4.2, we have considered the weights given by Case 2 .

Let us now formulate the Carleman inequality in Case $\mathbf{1}$ and Case $\mathbf{2}$.

Theorem 2.1. We assume that $\theta$ and $\varphi$ are given by (2.1) where $\lambda$ is fixed and large enough or by (2.4). In this last case, we assume that $\Gamma$ is such that (2.5) holds. Then, there exists $s_{0}>0$ and $C>0$ such that, for all $s \geq s_{0}:$

$$
\begin{aligned}
\int_{0}^{T} \int_{\Omega} e^{2 s \varphi}\left(\frac{1}{s \theta}\left|\partial_{t} z\right|^{2}+\frac{1}{s \theta}|\Delta z|^{2}+\right. & \left.s \theta|\nabla z|^{2}+s^{3} \theta^{3}|z|^{2}\right) \mathrm{d} x \mathrm{~d} t \\
& \leq C \int_{0}^{T} \int_{\Omega} e^{2 s \varphi}\left|\partial_{t} z-\Delta z\right|^{2} \mathrm{~d} x \mathrm{~d} t+C s \int_{0}^{T} \int_{\Gamma} e^{2 s \varphi} \theta|\nabla z \cdot n|^{2} \mathrm{~d} \gamma \mathrm{d} t
\end{aligned}
$$

for all $z \in H^{1}\left(0, T ; L^{2}(\Omega)\right) \cap L^{2}\left(0, T ; H^{2}(\Omega) \cap H_{0}^{1}(\Omega)\right)$.

Here and in all the paper, we denote by $C$ a positive constant which depends on $T$ and $\Omega, \lambda$ in Case $\mathbf{1}$ and $\rho$ in Case 2, unless specified otherwise where appropriated. The proof of this theorem is given in Appendix A. A consequence of Theorem 2.1 is the following lemma:

Lemma 2.2. Under the same assumptions as Theorem 2.1, there exist $s_{0}>0$ and $C>0$ such that, for all $s \geq s_{0}:$

$$
s \int_{\Omega} e^{2 s \varphi\left(T_{0}\right)}\left|z\left(T_{0}\right)\right|^{2} \mathrm{~d} x \leq C \int_{0}^{T} \int_{\Omega} e^{2 s \varphi}\left|\partial_{t} z-\Delta z\right|^{2} \mathrm{~d} x \mathrm{~d} t+C s \int_{0}^{T} \int_{\Gamma} e^{2 s \varphi} \theta|\nabla z \cdot n|^{2} \mathrm{~d} \gamma \mathrm{d} t,
$$

for all $z \in H^{1}\left(0, T ; L^{2}(\Omega)\right) \cap L^{2}\left(0, T ; H^{2}(\Omega) \cap H_{0}^{1}(\Omega)\right)$.

Proof. We have

$$
\begin{aligned}
\int_{\Omega} e^{2 s \varphi\left(T_{0}\right)}\left|z\left(T_{0}\right)\right|^{2} \mathrm{~d} x & =\int_{0}^{T_{0}} \frac{\mathrm{d}}{\mathrm{d} t}\left(\int_{\Omega} e^{2 s \varphi}|z|^{2} \mathrm{~d} x\right) \mathrm{d} t=\int_{0}^{T_{0}} \int_{\Omega} \partial_{t}\left(e^{2 s \varphi}|z|^{2}\right) \mathrm{d} x \mathrm{~d} t \\
& =\int_{0}^{T_{0}} \int_{\Omega} e^{2 s \varphi}\left(2 z\left(s \theta^{1 / 2} \frac{1}{s \theta^{1 / 2}}\right) \partial_{t} z+2 s \partial_{t} \varphi|z|^{2}\right) \mathrm{d} x \mathrm{~d} t \\
& \leq \int_{0}^{T_{0}} \int_{\Omega} e^{2 s \varphi} \frac{1}{s^{2} \theta}\left|\partial_{t} z\right|^{2} \mathrm{~d} x \mathrm{~d} t+\int_{0}^{T_{0}} \int_{\Omega} e^{2 s \varphi}\left(s^{2} \theta+2 s\left|\partial_{t} \varphi\right|\right)|z|^{2} \mathrm{~d} x \mathrm{~d} t \\
& \leq \frac{C}{s}\left[\int_{0}^{T_{0}} \int_{\Omega} e^{2 s \varphi} \frac{1}{s \theta}\left|\partial_{t} z\right|^{2} \mathrm{~d} x \mathrm{~d} t+\int_{0}^{T_{0}} \int_{\Omega} e^{2 s \varphi} s^{3} \theta^{2}|z|^{2} \mathrm{~d} x \mathrm{~d} t\right]
\end{aligned}
$$


where we have used that $\left|\partial_{t} \varphi\right| \leq C \theta^{2}$. Thus, the result follows from (2.6).

Remark 2.3. To better design Carleman weights for numerical purposes, it would be interesting to make a comprehensive comparison between different possible choices of Carleman weights for the heat equation. In particular, in such a study which is beyond the scope of our paper, it would be necessary to spell the lower bound on $s$ in the associated Carleman inequality.

Remark 2.4. We could have considered other kinds of boundary data by completing the first equation of (1.1) with Neumann conditions instead of Dirichlet conditions and by replacing the first measurement in (1.2) by a measurement on a part of the boundary of $u$ itself. In this case, following [11, 13], we still have a Carleman inequality with the classical weights (2.1) and we can still prove the global convergence of the numerical method. For the numerical tests, it would be interesting to see if we can get a Carleman inequality with weights similar to the ones of Case 2.

\subsection{Regularity result}

Let us give a regularity result for problem (1.1). The proof of this result is presented in Appendix B.

Proposition 2.5. Assume that $u_{\circ} \in H^{3}(\Omega), \sigma \in L^{\infty}(\Omega), h \in H^{1}\left(0, T ; L^{2}(\Omega)\right)$ and $g \in H^{1}\left(0, T ; H^{3 / 2}(\partial \Omega)\right) \cap$ $H^{2}\left(0, T ; H^{1 / 2}(\partial \Omega)\right)$. Moreover, we assume that $h(0, \cdot)=0$ in $\Omega$.

Then the solution $u$ of (1.1) belongs to

$$
u \in C^{1}\left(0, T ; H^{1}(\Omega)\right) \cap H^{2}\left(0, T ; L^{2}(\Omega)\right) \cap H^{1}\left(0, T ; H^{2}(\Omega)\right)
$$

with the estimate

$$
\begin{aligned}
& \|u\|_{C^{1}\left(0, T ; H^{1}(\Omega)\right)}+\|u\|_{H^{2}\left(0, T ; L^{2}(\Omega)\right)}+\|u\|_{H^{1}\left(0, T ; H^{2}(\Omega)\right)} \\
& \leq C\left(\|\sigma\|_{L^{\infty}(\Omega)}+\|\sigma\|_{L^{\infty}(\Omega)}^{p}\right)\left(\|h\|_{H^{1}\left(0, T ; L^{2}(\Omega)\right)}+\|h\|_{H^{1}\left(0, T ; L^{2}(\Omega)\right)}^{p}\right)+C\left(\left\|u_{\circ}\right\|_{H^{3}(\Omega)}+\left\|u_{\circ}\right\|_{H^{3}(\Omega)}^{p}\right) \\
& \quad+C\left(\|g\|_{H^{1}\left(0, T ; H^{3 / 2}(\partial \Omega)\right) \cap H^{2}\left(0, T ; L^{2}(\partial \Omega)\right)}+\|g\|_{H^{1}\left(0, T ; H^{3 / 2}(\partial \Omega)\right) \cap H^{2}\left(0, T ; H^{1 / 2}(\partial \Omega)\right)}^{p}\right)
\end{aligned}
$$

where the power $p>1$ is a fixed integer and $C$ only depends on $T$ and $\Omega$.

Let us note that, in the above proposition, the regularity assumed for $g$ is not optimal, it would indeed be sufficient to assume that $g \in H^{1}\left(0, T ; H^{3 / 2}(\partial \Omega)\right) \cap H^{2}\left(0, T ; H^{\kappa}(\partial \Omega)\right)$ with $\kappa>0$ (see [24], Chap. 1, Sect. 9.2). In this result, if we do not make the assumption that $h(0, \cdot)=0$ in $\Omega$, it is necessary to assume that $\sigma$ belongs to $H^{1}(\Omega)$ (since we need an initial condition in $H^{1}(\Omega)$ for the problem satisfied by $\partial_{t} u$ ). But this additional regularity assumption on $\sigma$ leads to difficulties in the construction of the iterations in Algorithm 3.2.

\subsection{Stability inequality}

In this paragraph, we state a Lipschitz stability inequality for our inverse problem. This result asserts in particular that the unknown $\sigma$ is identifiable from the measurements given by (1.2). It is obtained thanks to a direct application of Bukhgeim-Klibanov method [5] and relies on the Carleman inequality given by Theorem 2.1 and the regularity result given by Proposition 2.5. We do not give the proof here and refer to [15] for a closely related result.

Proposition 2.6. We assume that $u_{\circ} \in H^{3}(\Omega), g \in H^{1}\left(0, T ; H^{3 / 2}(\partial \Omega)\right) \cap H^{2}\left(0, T ; H^{1 / 2}(\partial \Omega)\right)$ and $h \in$ $H^{1}\left(0, T ; L^{\infty}(\Omega)\right)$ is such that $h(0, \cdot)=0$ in $\Omega$ and $\left|h\left(T_{0}, \cdot\right)\right| \geq \beta>0$ in $\Omega$. We consider $\sigma_{1}$ and $\sigma_{2}$ in $L^{\infty}(\Omega)$ which satisfy (1.3). Then, for $i=1,2$, if we denote by $u_{i}$ the solution of (1.1) associated to $\sigma_{i}$, we have the following inequality: there exists $C>0$ such that

$$
\left\|\sigma_{1}-\sigma_{2}\right\|_{L^{2}(\Omega)} \leq C\left(\left\|u_{1}\left(T_{0}\right)-u_{2}\left(T_{0}\right)\right\|_{H^{2}(\Omega)}+\left\|\nabla\left(u_{1}-u_{2}\right) \cdot n\right\|_{H^{1}\left(0, T ; L^{2}(\Gamma)\right)}\right) .
$$




\section{NUMERICAL RECONSTRUCTION METHOD AND THEORETICAL STUDY}

\subsection{Presentation of the algorithm and convergence}

In this subsection, we construct a sequence $\left(\sigma_{k}\right)_{k \in \mathbb{N}}$ which approximates the unknown $\sigma$ and we state the convergence of this sequence. We make the following assumptions:

Hypothesis 3.1. $\quad-u_{\circ} \in H^{3}(\Omega)$ and $g \in H^{1}\left(0, T ; H^{3 / 2}(\partial \Omega)\right) \cap H^{2}\left(0, T ; H^{1 / 2}(\partial \Omega)\right)$.

$-\sigma \in L^{\infty}(\Omega)$ satisfies $(1.3)$.

$-h$ satisfies

$$
h \in H^{1}\left(0, T ; L^{\infty}(\Omega)\right), \quad h(0, \cdot)=0 \text { in } \Omega
$$

and

$$
\left|h\left(T_{0}, \cdot\right)\right| \geq \beta>0 \quad \text { in } \Omega \text {. }
$$

- The weights $\theta$ and $\varphi$ are given by Case 1 or Case $\mathbf{2}$ described at the beginning of Section 2.1. In Case 1, the parameter $\lambda$ is fixed and large enough.

In our paper, we denote by $M$ an arbitrary constant which only depends on $T, \Omega, \sigma_{\max },\left\|u_{\circ}\right\|_{H^{3}(\Omega)}$, $\|h\|_{H^{1}\left(0, T ; L^{\infty}(\Omega)\right)}$ and $\|g\|_{H^{1}\left(0, T ; H^{3 / 2}(\partial \Omega)\right) \cap H^{2}\left(0, T ; H^{1 / 2}(\partial \Omega)\right)}$.

First, we initialize the sequence with $\sigma_{0}=0$ (or any guess such that $\left\|\sigma_{0}\right\|_{L^{\infty}(\Omega)} \leq \sigma_{\max }$ ).

Now, let us assume that we are at step $k$ and that we have constructed $\sigma_{k}$ which satisfies

$$
\left\|\sigma_{k}\right\|_{L^{\infty}(\Omega)} \leq \sigma_{\max }
$$

We denote by $u_{k}$ the solution of (1.1) associated to $\sigma_{k}$ and by $u_{\sigma}$ the solution of (1.1) associated to the unknown $\sigma$. Moreover, we set $v_{k}=u_{\sigma}-u_{k}$.

We then use Proposition 2.5 and we denote by $\bar{M}>0$ a fixed constant depending on $T, \Omega, \sigma_{\max },\left\|u_{\circ}\right\|_{H^{3}(\Omega)}$, $\|h\|_{H^{1}\left(0, T ; L^{2}(\Omega)\right)}$ and $\|g\|_{H^{1}\left(0, T ; H^{3 / 2}(\partial \Omega)\right) \cap H^{2}\left(0, T ; H^{1 / 2}(\partial \Omega)\right)}$ such that

$$
\left\|v_{k}\right\|_{C([0, T] \times \bar{\Omega})}+\left\|v_{k}\right\|_{C^{1}\left(0, T ; H^{1}(\Omega)\right)}+\left\|v_{k}\right\|_{H^{2}\left(0, T ; L^{2}(\Omega)\right)}+\left\|v_{k}\right\|_{H^{1}\left(0, T ; H^{2}(\Omega)\right)} \leq \bar{M} .
$$

The function $v_{k}$ is solution of

$$
\begin{cases}\partial_{t} v_{k}(t, x)-\Delta v_{k}(t, x)+v_{k}(t, x) q\left[v_{k}, u_{k}\right](t, x)=\left(\sigma(x)-\sigma_{k}(x)\right) h(t, x), & (t, x) \in(0, T) \times \Omega \\ v_{k}(t, x)=0, & (t, x) \in(0, T) \times \partial \Omega \\ v_{k}(0, x)=0, & x \in \Omega,\end{cases}
$$

where we have set $q[v, u]=3 u^{2}+3 u v+v^{2}$. Let us differentiate the equation with respect to time. We introduce $w_{k}=\partial_{t} v_{k}$ which satisfies:

$$
\begin{cases}\partial_{t} w_{k}(t, x)-\Delta w_{k}(t, x)+w_{k}(t, x) q\left[v_{k}, u_{k}\right](t, x)+v_{k}(t, x) \partial_{t}\left(q\left[v_{k}, u_{k}\right]\right)(t, x)=f_{k}(t, x), & (t, x) \in(0, T) \times \Omega, \\ w_{k}(t, x)=0, & (t, x) \in(0, T) \times \partial \Omega \\ w_{k}(0, x)=0, & x \in \Omega,\end{cases}
$$

where, for all $(t, x) \in(0, T) \times \Omega$,

$$
f_{k}(t, x)=\left(\sigma(x)-\sigma_{k}(x)\right) \partial_{t} h(t, x) .
$$


Let us now explain the core idea of the numerical method that we will introduce below. We notice in (3.5) that

$$
w_{k}\left(T_{0}, x\right)=\partial_{t} v_{k}\left(T_{0}, x\right)=\Delta v_{k}\left(T_{0}, x\right)-v_{k}\left(T_{0}, x\right) q\left[v_{k}, u_{k}\right]\left(T_{0}, x\right)+\left(\sigma(x)-\sigma_{k}(x)\right) h\left(T_{0}, x\right), \quad x \in \Omega .
$$

Hence, if $w_{k}\left(T_{0}, \cdot\right)$ was known, then $\sigma$ could be directly computed, because we assume that $h\left(T_{0}, \cdot\right)$ satisfies $(3.2)$ and the other terms in (3.8) are given observations thanks to (1.2). However, since $f_{k}$ defined in (3.7) depends on $\sigma, w_{k}$ is unknown. Thus, the idea is to use $Z_{k}$ obtained via the minimization step (Step 2) of Algorithm 3.2 as a proxy for $w_{k}$. In Section 3.4, we will estimate the discrepancy between $Z_{k}$ and $w_{k}$ (both seen as minimizers of functionals) with respect to $f_{k}$.

For the constant $\bar{M}>0$ introduced in estimate (3.4), we consider the following function:

$$
\begin{aligned}
T_{\bar{M}}: & \mathbb{R} \longrightarrow \mathbb{R} \\
& X \longmapsto X \Phi\left(\frac{X}{\bar{M}}\right),
\end{aligned}
$$

where $\Phi \in C_{0}^{2}(\mathbb{R})$ is such that $0 \leq \Phi \leq 1$ and

$$
\Phi(X)= \begin{cases}1, & \text { if }|X| \leq 1 \\ 0, & \text { if }|X| \geq 2\end{cases}
$$

The properties satisfied by $T_{\bar{M}}$ are given in Section 3.2. For any $\mu$ in $L^{2}((0, T) \times \Gamma)$ and for $s$ large enough, we introduce the functional $J_{0, k}[\mu]$ by

$$
J_{0, k}[\mu](z)=\frac{1}{2} \int_{0}^{T} \int_{\Omega} e^{2 s \varphi}\left|P_{k} z\right|^{2} \mathrm{~d} x \mathrm{~d} t+\frac{s}{2} \int_{0}^{T} \int_{\Gamma} e^{2 s \varphi} \theta|\nabla z \cdot n-\mu|^{2} \mathrm{~d} \gamma \mathrm{d} t
$$

with

$$
P_{k} z=\partial_{t} z-\Delta z+3\left(u_{k}\right)^{2} z+6 \partial_{t} u_{k} u_{k} T_{\bar{M}}(y)+3 \partial_{t} u_{k} T_{\bar{M}}(y)^{2}+6 u_{k} z T_{\bar{M}}(y)+3 z T_{\bar{M}}(y)^{2}
$$

where

$$
y(t, x)=v_{k}\left(T_{0}, x\right)+\int_{T_{0}}^{t} z\left(t^{\prime}, x\right) \mathrm{d} t^{\prime}, \quad(t, x) \in(0, T) \times \Omega .
$$

By this way, since $v_{k}$ satisfies $(3.4), T_{\bar{M}}\left(v_{k}\right)=v_{k}$ and $P_{k}\left(w_{k}\right)$ corresponds to the left hand side of the first equation of (3.6).

We consider the functional $J_{0, k}[\mu]$ on the function space

$$
\begin{aligned}
\widetilde{E}=\left\{z: e^{s \varphi}\right. & \left(\partial_{t} z-\Delta z\right) \in L^{2}((0, T) \times \Omega), e^{s \varphi} \theta^{1 / 2} \nabla z \cdot n \in L^{2}((0, T) \times \Gamma), \\
& \left.\times e^{s \varphi} \theta^{3 / 2} z \in L^{2}((0, T) \times \Omega), e^{s \varphi} \theta^{-1 / 2} z \in H^{1}\left(0, T ; L^{2}(\Omega)\right) \cap L^{2}\left(0, T ; H^{2}(\Omega) \cap H_{0}^{1}(\Omega)\right)\right\}
\end{aligned}
$$

endowed with its natural norm.

The next iteration $\sigma_{k+1}$ is defined by following four steps: 
Algorithm 3.2. Iteration: From $k$ to $k+1$

- Step 1 - We set $\mu_{k}=\partial_{t}\left(m-\nabla u_{k} \cdot n\right)$ on $(0, T) \times \Gamma$, where $m$ is the measurement defined in (1.2) and $u_{k}$ is the solution of (1.1) associated to $\sigma_{k}$.

- Step 2 - We denote by $Z_{k}$ a critical point of $J_{0, k}\left[\mu_{k}\right]$ in $\widetilde{E}$.

- Step 3 - We set

$$
\widetilde{\sigma}_{k+1}(x)=\sigma_{k}(x)+\frac{Z_{k}\left(T_{0}, x\right)-\Delta v_{k}\left(T_{0}, x\right)+v_{k}\left(T_{0}, x\right) q\left[v_{k}, u_{k}\right]\left(T_{0}, x\right)}{h\left(T_{0}, x\right)}, \quad x \in \Omega .
$$

- Step 4 - At last, we define

$$
\sigma_{k+1}=\Pi_{\sigma_{\max }}\left(\widetilde{\sigma}_{k+1}\right)
$$

where $\Pi_{\sigma_{\max }}$ is given by

$$
\Pi_{\sigma_{\max }}(\sigma)=\left\{\begin{array}{cc}
\sigma, & \text { if }|\sigma| \leq \sigma_{\max } \\
\operatorname{sign}(\sigma) \sigma_{\max }, & \text { otherwise. }
\end{array}\right.
$$

Remark 3.3. Let us give some comments regarding the different steps of Algorithm 3.2.

- According to Lemma 3.7, $J_{0, k}\left[\mu_{k}\right]$ (defined in (3.11)) admits a global minimizer in $\widetilde{E}$ if $s$ is large enough. Hence, this result in particular ensures the existence of a critical point which is needed in Step 2 of the algorithm. For the numerical implementation of this step, we refer to Remark 4.2. Let us also notice that, as the functional $J_{0, k}\left[\mu_{k}\right], Z_{k}$ depends on $s$ but we drop this dependence to avoid heavy notations.

- In Step 3, $\widetilde{\sigma}_{k+1}$ is well-defined because $h$ satisfies the positivity condition $(3.2)$ and $h\left(T_{0}, \cdot\right)$ belongs to $L^{2}(\Omega)$. Moreover, in this expression, $v_{k}\left(T_{0}, \cdot\right)$ is known and given by $v_{k}\left(T_{0}, \cdot\right)=r-u_{k}\left(T_{0}, \cdot\right)$ where $r$ is the measurement defined in (1.2). Since $u_{k}\left(T_{0}\right)$ and $v_{k}\left(T_{0}\right)$ belong to $H^{2}(\Omega)$ and $Z_{k}\left(T_{0}\right)$ belongs to $L^{2}(\Omega)$, $\widetilde{\sigma}_{k+1}$ belongs to $L^{2}(\Omega)$.

- Step 4 is needed to ensure that $\sigma_{k+1}$ satisfies (3.3) at step $k+1$.

Now we state the main theoretical result which gives the global linear convergence in the weighted $L^{2}$-norm of the sequence $\left(\sigma_{k}\right)_{k \in \mathbb{N}}$ :

Theorem 3.4. Under Hypotheses 3.1, there exist $s_{0}>0$ and $M>0$ such that for all $s \geq s_{0}$, for all $k \in \mathbb{N}$

$$
\int_{\Omega} e^{2 s \varphi\left(T_{0}\right)}\left|\sigma_{k+1}-\sigma\right|^{2} \mathrm{~d} x \leq \frac{M}{s} \int_{\Omega} e^{2 s \varphi\left(T_{0}\right)}\left|\sigma_{k}-\sigma\right|^{2} \mathrm{~d} x
$$

Thus, for s large enough, $\left(\sigma_{k}\right)_{k \in \mathbb{N}}$ tends to $\sigma$ when $k$ goes to $+\infty$.

This theorem will be proved in Section 3.4.

Remark 3.5. Let us notice that our method may also be applied to the identification of a source in the simpler case of the linear heat equation. In this case, the inverse problem is linear and thus the properties of our method (in particular the global convergence) are much more classical. If we simply consider the least square method, the functional is quadratic and, thanks to the stability estimate, we can prove that this functional is strongly convex. Thus, a classical gradient descent method globally converges and it is not necessary to introduce our algorithm which is more complex. 


\subsection{Properties satisfied by the function $\boldsymbol{T}_{\bar{M}}$}

Proposition 3.6. The function $T_{\bar{M}}$ defined by (3.9) belongs to $C_{0}^{2}(\mathbb{R})$ satisfies the following properties:

a) For all $X \in \mathbb{R}$,

$$
\left|T_{\bar{M}}(X)\right| \leq 2 \bar{M}
$$

b) There exists $L>0$ such that

$$
\left|T_{\bar{M}}^{\prime}(X)\right| \leq L \chi_{[-2 \bar{M}, 2 \bar{M}]}(X), \quad \forall X \in \mathbb{R},
$$

where $\chi_{A}$ is the characteristic function of a set $A$.

c) For all $X_{1}, X_{2} \in \mathbb{R}$,

$$
\left|T_{\bar{M}}\left(X_{1}\right)-T_{\bar{M}}\left(X_{2}\right)\right| \leq L\left|X_{1}-X_{2}\right|,
$$

which implies in particular that $T_{\bar{M}}$ is a Lipschitz operator.

d) There exists $C>0$ such that, for all $X_{1}, X_{2} \in \mathbb{R}$,

$$
\left|T_{\bar{M}}^{\prime}\left(X_{1}\right)-T_{\bar{M}}^{\prime}\left(X_{2}\right)\right| \leq C\left|X_{1}-X_{2}\right|,
$$

Proof.

a) For $X \in \mathbb{R}$, we have

$$
\left|T_{\bar{M}}(X)\right|=\left|X \Phi\left(\frac{X}{\bar{M}}\right)\right|=\left\{\begin{array}{ll}
\leq|X|, & \text { if }|X| \leq 2 \bar{M} \\
=0, & \text { if }|X|>2 \bar{M}
\end{array}\right\} \leq 2 \bar{M} .
$$

b) By definition (3.9), $T_{\bar{M}}^{\prime}(X)=0$ for all $|X| \geq 2 \bar{M}$. Moreover, for all $|X| \leq 2 \bar{M}$, we have

$$
\begin{aligned}
\left|T_{\bar{M}}^{\prime}(X)\right| & =\left|\Phi\left(\frac{X}{\bar{M}}\right)+\frac{X}{\bar{M}} \Phi^{\prime}\left(\frac{X}{\bar{M}}\right)\right| \\
& \leq 1+2\left\|\Phi^{\prime}\right\|_{C_{0}(\mathbb{R})} .
\end{aligned}
$$

c) This is a direct consequence of (3.17) and the mean value inequality.

d) By the same arguments as in b), we show that

$$
\left|T_{\bar{M}}^{\prime \prime}(X)\right| \leq L \chi_{[-2 \bar{M}, 2 \bar{M}]}(X), \quad \forall X \in \mathbb{R} .
$$

Hence, (3.19) is a direct consequence of (3.20) and the mean value inequality.

\subsection{Properties satisfied by $J_{0, k}[\mu]$}

The following lemma ensures the existence of a global minimizer of $J_{0, k}[\mu]$ in $\widetilde{E}$. This result in particular ensures the existence of a critical point $Z_{k}$ introduced at Step 2 in Algorithm 3.2.

Lemma 3.7. Let $\mu$ be given in $L^{2}((0, T) \times \Gamma)$ and assume that Hypotheses 3.1 hold. There exists $s_{0}>0$ which depends on $T, \Omega, \sigma_{\max },\left\|u_{\circ}\right\|_{H^{3}(\Omega)},\|h\|_{H^{1}\left(0, T ; L^{\infty}(\Omega)\right)}$, and $\|g\|_{H^{1}\left(0, T ; H^{3 / 2}(\partial \Omega)\right) \cap H^{2}\left(0, T ; H^{1 / 2}(\partial \Omega)\right)}$ such that for all $s \geq s_{0}$ and for all $k \in \mathbb{N}$, the functional $J_{0, k}[\mu]$ defined by (3.11) admits a global minimizer in $\widetilde{E}$. 
Proof. According to Proposition 2.5, there exists a constant $M>0$ such that

$$
\left\|u_{k}\right\|_{C^{1}\left(0, T ; H^{1}(\Omega)\right)}+\left\|u_{k}\right\|_{H^{2}\left(0, T ; L^{2}(\Omega)\right)}+\left\|u_{k}\right\|_{H^{1}\left(0, T ; H^{2}(\Omega)\right)} \leq M
$$

Using this estimate and (3.18), we have the continuity of $J_{0, k}[\mu]$ in $\widetilde{E}$. Moreover, since $J_{0, k}[\mu]$ is positive, it admits an infimum in $\widetilde{E}$ and we can introduce a sequence $\left(z_{n}\right)_{n \in \mathbb{N}}$ such that

$$
J_{0, k}[\mu]\left(z_{n}\right) \underset{n \rightarrow+\infty}{\longrightarrow} \inf _{z \in \widetilde{E}} J_{0, k}[\mu](z)
$$

Let us study the convergence properties of the sequence $\left(z_{n}\right)_{n \in \mathbb{N}}$. First, we notice that we can write $P_{k} z_{n}$ under the form

$$
P_{k} z_{n}=\partial_{t} z_{n}-\Delta z_{n}+r_{k, n} z_{n}+s_{k, n}
$$

where $r_{k, n}$ and $s_{k, n}$ only depend on $u_{k}, \partial_{t} u_{k}$ and $T_{\bar{M}}\left(y_{n}\right)$. Using inequality (3.21) and the property (3.16), we deduce that $r_{k, n}$ is bounded in $L^{\infty}((0, T) \times \Omega)$ and $s_{k, n}$ is bounded in $L^{2}((0, T) \times \Omega)$ by some constant $M$. Hence, writing that

$$
\left|P_{k} z_{n}\right|^{2} \geq \frac{1}{2}\left|\partial_{t} z_{n}-\Delta z_{n}\right|^{2}-2\left|r_{k, n} z_{n}\right|^{2}-2\left|s_{k, n}\right|^{2}
$$

we get

$$
\begin{aligned}
J_{0, k}[\mu]\left(z_{n}\right) \geq & \frac{1}{4} \int_{0}^{T} \int_{\Omega} e^{2 s \varphi}\left|\partial_{t} z_{n}-\Delta z_{n}\right|^{2} \mathrm{~d} x \mathrm{~d} t-M \int_{0}^{T} \int_{\Omega} e^{2 s \varphi}\left|z_{n}\right|^{2} \mathrm{~d} x \mathrm{~d} t-M \\
& +\frac{s}{4} \int_{0}^{T} \int_{\Gamma} e^{2 s \varphi} \theta\left|\nabla z_{n} \cdot n\right|^{2} \mathrm{~d} \gamma \mathrm{d} t-\frac{s}{2} \int_{0}^{T} \int_{\Gamma} e^{2 s \varphi} \theta \mu^{2} \mathrm{~d} \gamma \mathrm{d} t .
\end{aligned}
$$

According to the Carleman inequality given by (2.6) and using the fact that $s e^{2 s \varphi} \theta \leq C$ in $(0, T) \times \Omega$ for the third term in the right hand side, we deduce that, for $s$ large enough,

$$
\int_{0}^{T} \int_{\Omega} e^{2 s \varphi}\left(\frac{1}{s \theta}\left|\partial_{t} z_{n}\right|^{2}+\frac{1}{s \theta}\left|\Delta z_{n}\right|^{2}+s \theta\left|\nabla z_{n}\right|^{2}+s^{3} \theta^{3}\left|z_{n}\right|^{2}\right) \mathrm{d} x \mathrm{~d} t \leq J_{0, k}[\mu]\left(z_{n}\right)+M+C\|\mu\|_{L^{2}((0, T) \times \Gamma)}^{2} .
$$

By construction of $\left(z_{n}\right)_{n \in \mathbb{N}}$, the sequence $\left(J_{0, k}[\mu]\left(z_{n}\right)\right)_{n \in \mathbb{N}}$ is bounded and thus the left hand side of this last inequality is bounded. According to the definitions of $\theta$ and $\varphi$ which are given by (2.1) or by (2.4), we have in $(0, T) \times \Omega$

$$
\left|\partial_{t} \theta\right|+\left|\partial_{t} \varphi\right| \leq C \theta^{2} \text { and }|\nabla \theta|+|\nabla \varphi|+\left|D^{2} \theta\right|+\left|D^{2} \varphi\right| \leq C \theta
$$

and thus $\left(e^{s \varphi} \theta^{-1 / 2} z_{n}\right)_{n \in \mathbb{N}}$ is bounded in $H^{1}\left(0, T ; L^{2}(\Omega)\right) \cap L^{2}\left(0, T ; H^{2}(\Omega)\right)$. We deduce that, $\left(e^{s \varphi} \theta^{-1 / 2} z_{n}\right)_{n \in \mathbb{N}}$ weakly converges to some element in $H^{1}\left(0, T ; L^{2}(\Omega)\right) \cap L^{2}\left(0, T ; H^{2}(\Omega)\right.$ ) that we denote $e^{s \varphi} \theta^{-1 / 2} \tilde{z}$ (all the convergence results given in this proof are valid up to a subsequence but we do not specify it in order to lighten the writing).

Moreover, since $H^{1}\left(0, T ; L^{2}(\Omega)\right) \cap L^{2}\left(0, T ; H^{2}(\Omega)\right)$ is compactly embedded in $L^{2}((0, T) \times \Omega)$,

$$
e^{s \varphi} \theta^{-1 / 2} z_{n} \rightarrow e^{s \varphi} \theta^{-1 / 2} \tilde{z} \text { in } L^{2}((0, T) \times \Omega)
$$


and, by identification of the limit, since $\theta^{-1}$ belongs to $L^{\infty}((0, T) \times \Omega)$, we also have

$$
e^{s \varphi} \theta^{3 / 2} z_{n} \rightarrow e^{s \varphi} \theta^{3 / 2} \tilde{z} \text { weakly in } L^{2}((0, T) \times \Omega)
$$

and

$$
e^{s \varphi} \theta^{1 / 2} \nabla z_{n} \rightarrow e^{s \varphi} \theta^{1 / 2} \nabla \tilde{z} \text { weakly in } L^{2}\left(0, T ; H^{1}(\Omega)\right) .
$$

Let us now prove that $\lim _{n \rightarrow+\infty} J_{0, k}[\mu]\left(z_{n}\right)=J_{0, k}[\mu](\tilde{z})$ which will imply that $\tilde{z}$ minimizes $J_{0, k}[\mu]$. Since $\left(J_{0, k}[\mu]\left(z_{n}\right)\right)_{n \in \mathbb{N}}$ is bounded, $\left(e^{s \varphi} \theta^{1 / 2}\left(\nabla z_{n} \cdot n\right)\right)_{n \in \mathbb{N}}$ weakly converges in $L^{2}((0, T) \times \Gamma)$ and $\left(e^{s \varphi} P_{k} z_{n}\right)_{n \in \mathbb{N}}$ weakly converges in $L^{2}((0, T) \times \Omega)$ and it is sufficient to identify their weak limits. The fact that

$$
e^{s \varphi} \theta^{1 / 2}\left(\nabla z_{n} \cdot n\right) \rightarrow e^{s \varphi} \theta^{1 / 2}(\nabla \tilde{z} \cdot n) \text { weakly in } L^{2}((0, T) \times \Gamma)
$$

directly comes from (3.24). To identify the limit of $\left(e^{s \varphi} P_{k} z_{n}\right)_{n \in \mathbb{N}}$, we will prove that

$$
e^{s \varphi} \theta^{-1 / 2} P_{k} z_{n} \rightarrow e^{s \varphi} \theta^{-1 / 2} P_{k} \tilde{z} \text { weakly in } L^{2}((0, T) \times \Omega) .
$$

We first consider in the definition (3.12) of $P_{k}$ the three first terms which correspond to the linear part. The weak convergence of $\left(e^{s \varphi} \theta^{-1 / 2} z_{n}\right)_{n \in \mathbb{N}}$ to $e^{s \varphi} \theta^{-1 / 2} \tilde{z}$ in $H^{1}\left(0, T ; L^{2}(\Omega)\right) \cap L^{2}\left(0, T ; H^{2}(\Omega)\right)$ implies that

$$
e^{s \varphi} \theta^{-1 / 2}\left(\partial_{t} z_{n}-\Delta z_{n}+3\left(u_{k}\right)^{2} z_{n}\right) \rightarrow e^{s \varphi} \theta^{-1 / 2}\left(\partial_{t} \tilde{z}-\Delta \tilde{z}+3\left(u_{k}\right)^{2} \tilde{z}\right) \quad \text { weakly in } L^{2}((0, T) \times \Omega) .
$$

Now we define, for all $t \in(0, T)$

$$
y_{n}(t)=v_{k}\left(T_{0}\right)+\int_{T_{0}}^{t} z_{n}\left(t^{\prime}\right) \mathrm{d} t^{\prime} \quad \text { and } \quad \tilde{y}(t)=v_{k}\left(T_{0}\right)+\int_{T_{0}}^{t} \tilde{z}\left(t^{\prime}\right) \mathrm{d} t^{\prime}
$$

For the other terms in the operator $P_{k}$, let us first prove that $\left(e^{s \varphi} \theta^{-1 / 2} T_{\bar{M}}\left(y_{n}\right)\right)_{n \in \mathbb{N}}$ strongly converges to $e^{s \varphi} \theta^{-1 / 2} T_{\bar{M}}(\tilde{y})$ in $L^{\infty}\left(0, T ; L^{2}(\Omega)\right)$. To do so, we observe that

$$
\begin{aligned}
\int_{\Omega} e^{2 s \varphi} \theta^{-1 / 2}\left|y_{n}-\tilde{y}\right|^{2} \mathrm{~d} x & =\int_{\Omega} e^{2 s \varphi} \theta^{-1 / 2}\left|\int_{T_{0}}^{t}\left(z_{n}-\tilde{z}\right)\left(t^{\prime}, x\right) \mathrm{d} t^{\prime}\right|^{2} \mathrm{~d} x \\
& \leq C \int_{\Omega} e^{2 s \varphi} \theta^{-1 / 2}\left|\int_{T_{0}}^{t}\right| z_{n}-\left.\tilde{z}\right|^{2}\left(t^{\prime}, x\right) \mathrm{d} t^{\prime} \mid \mathrm{d} x
\end{aligned}
$$

By definition (2.1) or (2.4) of $\varphi$ and $\theta$ and since $T_{0}=\frac{T}{2}$, we have, for all $t^{\prime}$ between $T_{0}$ and $t$, for all $x \in \Omega$

$$
\varphi(t, x) \leq \varphi\left(t^{\prime}, x\right) \text { and } \theta(t, x) \geq \theta\left(t^{\prime}, x\right)
$$

This implies that

$$
\left\|e^{s \varphi} \theta^{-1 / 2}\left(y_{n}-\tilde{y}\right)\right\|_{L^{\infty}\left(0, T ; L^{2}(\Omega)\right)}^{2} \leq C \int_{0}^{T} \int_{\Omega} e^{2 s \varphi} \theta^{-1 / 2}\left|z_{n}-\tilde{z}\right|^{2} \mathrm{~d} x \mathrm{~d} t .
$$


Thus, according to $(3.23),\left(e^{s \varphi} \theta^{-1 / 2} y_{n}\right)_{n \in \mathbb{N}}$ strongly converges to $e^{s \varphi} \theta^{-1 / 2} \tilde{y}$ in $L^{\infty}\left(0, T ; L^{2}(\Omega)\right)$ and since $T_{\bar{M}}$ satisfies (3.18), this implies that

$$
e^{s \varphi} \theta^{-1 / 2} T_{\bar{M}}\left(y_{n}\right) \rightarrow e^{s \varphi} \theta^{-1 / 2} T_{\bar{M}}(\tilde{y}) \quad \text { in } L^{\infty}\left(0, T ; L^{2}(\Omega)\right) .
$$

We can now study the limit of the remaining terms of $e^{s \varphi} P_{k} z_{n}$ when $n$ tends to $+\infty$ : using (3.16), (3.21) and (3.28), we have

$$
e^{s \varphi} \theta^{-1 / 2} \partial_{t} u_{k} u_{k} T_{\bar{M}}\left(y_{n}\right) \rightarrow e^{s \varphi} \theta^{-1 / 2} \partial_{t} u_{k} u_{k} T_{\bar{M}}(\tilde{y}) \quad \text { in } L^{2}((0, T) \times \Omega)
$$

and

$$
e^{s \varphi} \theta^{-1 / 2} \partial_{t} u_{k} T_{\bar{M}}\left(y_{n}\right)^{2} \rightarrow e^{s \varphi} \theta^{-1 / 2} \partial_{t} u_{k} T_{\bar{M}}(\tilde{y})^{2} \quad \text { in } L^{2}((0, T) \times \Omega) .
$$

Let us now prove that

$$
e^{s \varphi} \theta^{-1 / 2} u_{k} z_{n} T_{\bar{M}}\left(y_{n}\right) \rightarrow e^{s \varphi} \theta^{-1 / 2} u_{k} \tilde{z} T_{\bar{M}}(\tilde{y}) \quad \text { in } L^{2}((0, T) \times \Omega) .
$$

The strong convergence (3.23) of $\left(e^{s \varphi} \theta^{-1 / 2} z_{n}\right)_{n \in \mathbb{N}}$ implies the almost everywhere convergence of $\left(z_{n}\right)_{n \in \mathbb{N}}$ to $\tilde{z}$ and the existence of a function $z_{b}$ in $L^{2}((0, T) \times \Omega)$ such that, for all $n \in \mathbb{N}$

$$
\left|e^{s \varphi} \theta^{-1 / 2} z_{n}\right| \leq z_{b}
$$

Moreover, the strong convergence of $\left(e^{s \varphi} \theta^{-1 / 2} y_{n}\right)_{n \in \mathbb{N}}$ in $L^{2}((0, T) \times \Omega)$ implies the almost everywhere convergence of $\left(y_{n}\right)_{n \in \mathbb{N}}$ to $\tilde{y}$. Thus, we deduce that

$$
e^{s \varphi} \theta^{-1 / 2} u_{k} z_{n} T_{\bar{M}}\left(y_{n}\right) \rightarrow e^{s \varphi} \theta^{-1 / 2} u_{k} \tilde{z} T_{\bar{M}}(\tilde{y}) \text { a.e. }
$$

and

$$
\left|e^{s \varphi} \theta^{-1 / 2} u_{k} z_{n} T_{\bar{M}}\left(y_{n}\right)\right| \leq M z_{b} .
$$

And these two properties imply (3.31) according to Lebesgue's dominated convergence theorem. At last, we use the same arguments to prove that

$$
e^{s \varphi} \theta^{-1 / 2} z_{n} T_{\bar{M}}\left(y_{n}\right)^{2} \rightarrow e^{s \varphi} \theta^{-1 / 2} u_{k} \tilde{z} T_{\bar{M}}(\tilde{y}) \quad \text { in } L^{2}((0, T) \times \Omega) .
$$

Finally, gathering (3.26) and (3.29) to (3.32), we obtain (3.25) and we conclude that $\tilde{z}$ is a minimizer of $J_{0, k}[\mu]$.

Due to the nonlinearities in our equation, we can not state the strong convexity of $J_{0, k}[\mu]$ in $\widetilde{E}$. Nevertheless, it is interesting to notice that the strong convexity property holds if we consider a smaller space than $\widetilde{E}$ including some boundedness hypotheses which allow to deal with the nonlinearities (similar results are obtained in [19])). We state this property in the following lemma:

Lemma 3.8. Let $C>0$ be fixed. We define

$$
E_{C}:=\left\{z \in H^{1}\left(0, T ; L^{2}(\Omega)\right) \cap L^{2}\left(0, T ; H^{2}(\Omega) \cap H_{0}^{1}(\Omega)\right),\|z\|_{L^{2}\left(0, T ; H^{2}(\Omega)\right)}+\|z\|_{H^{1}\left(0, T ; L^{2}(\Omega)\right)} \leq C\right\} .
$$

For $s_{0}$ large enough, $J_{0, k}[\mu]$ is strongly convex in $E_{C}$ for any $s \geq s_{0}$. 
This Lemma is proved in Appendix C. Contrary to the wave equation where the weights stay far from 0 (see [1], Sect. 4), our weights, as usual for the heat equation, vanish at 0 and $T$ and it is not clear that a minimizer of $J_{0, k}[\mu]$ in $\widetilde{E}$ will belong to $E_{C}$ for some $C>0$. Therefore, it is not possible to deduce the uniqueness in $\widetilde{E}$ from the strong convexity in $E_{C}$.

This convexity property is not used in the proof of Theorem 3.4 but it is an important property for the convergence of numerical minimization methods (we refer to Rem. 4.2 for a discussion on the numerical methods and the fact that the property shown here does not correspond exactly to the numerical framework).

\subsection{Proof of the convergence result stated in Theorem 3.4}

For $\mu_{k}=\partial_{t}\left(m-\nabla u_{k} \cdot n\right)$ on $(0, T) \times \Gamma$, we define the functional

$$
J_{k}\left[\mu_{k}\right](z)=\frac{1}{2} \int_{0}^{T} \int_{\Omega} e^{2 s \varphi}\left|P_{k} z-f_{k}\right|^{2} \mathrm{~d} x \mathrm{~d} t+\frac{s}{2} \int_{0}^{T} \int_{\Gamma} e^{2 s \varphi} \theta\left|\nabla z \cdot n-\mu_{k}\right|^{2} \mathrm{~d} \gamma \mathrm{d} t,
$$

where $P_{k}$ is given by (3.12) and $f_{k}$ is defined by (3.7). We notice that $w_{k}$, solution of the equation (3.6) minimizes $J_{k}\left[\mu_{k}\right]$ in $\widetilde{E}$. Indeed, according to $(3.4), T_{\bar{M}}\left(v_{k}\right)=v_{k}$ and this implies that $J_{k}\left[\mu_{k}\right]\left(w_{k}\right)=0$.

Let us now compute the Gâteaux derivative of $P_{k}$ at point $w$, for any $w \in \widetilde{E}$. Let $z \in \widetilde{E}$,

$$
\begin{aligned}
D P_{k}(w)(z)= & \lim _{\epsilon \rightarrow 0} \frac{P_{k}(w+\epsilon z)-P_{k}(w)}{\epsilon} \\
= & \partial_{t} z-\Delta z+3 z\left(\left(u_{k}\right)^{2}+2 u_{k} T_{\bar{M}}(v)+T_{\bar{M}}(v)^{2}\right) \\
& +6 T_{\bar{M}}^{\prime}(v) \bar{y}\left(\partial_{t} u_{k} u_{k}+\partial_{t} u_{k} T_{\bar{M}}(v)+u_{k} w+w T_{\bar{M}}(v)\right),
\end{aligned}
$$

where $v(t)=v_{k}\left(T_{0}\right)+\int_{T_{0}}^{t} w\left(t^{\prime}\right) \mathrm{d} t^{\prime}, \bar{y}(t)=\int_{T_{0}}^{t} z\left(t^{\prime}\right) \mathrm{d} t^{\prime}$

Then, $w_{k}$ satisfies the first order optimality condition given by

$$
\int_{0}^{T} \int_{\Omega} e^{2 s \varphi}\left(P_{k} w_{k}-f_{k}\right) D P_{k}\left(w_{k}\right)(z) \mathrm{d} x \mathrm{~d} t+s \int_{0}^{T} \int_{\Gamma} e^{2 s \varphi} \theta\left(\nabla w_{k} \cdot n-\mu_{k}\right)(\nabla z \cdot n) \mathrm{d} \gamma \mathrm{d} t=0, \quad \forall z \in \widetilde{E}
$$

Similarly, $Z_{k}$ satisfies the first order optimality condition

$$
\int_{0}^{T} \int_{\Omega} e^{2 s \varphi}\left(P_{k} Z_{k}\right) D P_{k}\left(Z_{k}\right)(z) \mathrm{d} x \mathrm{~d} t+s \int_{0}^{T} \int_{\Gamma} e^{2 s \varphi} \theta\left(\nabla Z_{k} \cdot n-\mu_{k}\right)(\nabla z \cdot n) \mathrm{d} \gamma \mathrm{d} t=0, \quad \forall z \in \widetilde{E} .
$$

Let us define $z_{k}=w_{k}-Z_{k}$. We compute the difference between (3.35) and (3.36) and take $z=z_{k}$. We get

$$
\begin{aligned}
& \int_{0}^{T} \int_{\Omega} e^{2 s \varphi}\left(\left(P_{k} w_{k}-P_{k} Z_{k}\right) D P_{k}\left(Z_{k}\right)\left(z_{k}\right)+P_{k} w_{k}\left(D P_{k}\left(w_{k}\right)\left(z_{k}\right)-D P_{k}\left(Z_{k}\right)\left(z_{k}\right)\right)\right) \mathrm{d} x \mathrm{~d} t \\
& +s \int_{0}^{T} \int_{\Gamma} e^{2 s \varphi} \theta\left|\nabla z_{k} \cdot n\right|^{2} \mathrm{~d} \gamma \mathrm{d} t=\int_{0}^{T} \int_{\Omega} e^{2 s \varphi} f_{k} D P_{k}\left(w_{k}\right)\left(z_{k}\right) \mathrm{d} x \mathrm{~d} t .
\end{aligned}
$$

This implies that

$$
\begin{aligned}
& \int_{0}^{T} \int_{\Omega} e^{2 s \varphi}\left(P_{k} w_{k}-P_{k} Z_{k}\right) D P_{k}\left(Z_{k}\right)\left(z_{k}\right) \mathrm{d} x \mathrm{~d} t+s \int_{0}^{T} \int_{\Gamma} e^{2 s \varphi} \theta\left|\nabla z_{k} \cdot n\right|^{2} \mathrm{~d} x \mathrm{~d} t \leq \int_{0}^{T} \int_{\Omega} e^{2 s \varphi}\left|f_{k}\right|^{2} \mathrm{~d} x \mathrm{~d} t \\
& +\frac{1}{4} \int_{0}^{T} \int_{\Omega} e^{2 s \varphi}\left|D P_{k}\left(w_{k}\right)\left(z_{k}\right)\right|^{2} \mathrm{~d} x \mathrm{~d} t+\int_{0}^{T} \int_{\Omega} e^{2 s \varphi}\left|P_{k} w_{k}\right|\left|D P_{k}\left(w_{k}\right)\left(z_{k}\right)-D P_{k}\left(Z_{k}\right)\left(z_{k}\right)\right| \mathrm{d} x \mathrm{~d} t .
\end{aligned}
$$


For what follows, we define, for all $t \in(0, T)$

$$
Y_{k}(t, \cdot)=v_{k}\left(T_{0}, \cdot\right)+\int_{T_{0}}^{t} Z_{k}\left(t^{\prime}, \cdot\right) \mathrm{d} t^{\prime} \quad \text { and } \quad \bar{y}_{k}(t, \cdot)=v_{k}(t, \cdot)-Y_{k}(t, \cdot)=\int_{T_{0}}^{t} z_{k}\left(t^{\prime}, \cdot\right) \mathrm{d} t^{\prime} .
$$

We will estimate separately the different terms of this inequality. We divide the computations in several steps.

- Step 1. Let us first find a lower bound for the first term in the left-hand side of (3.38).

$$
\begin{aligned}
P_{k} w_{k}-P_{k} Z_{k}= & \partial_{t} z_{k}-\Delta z_{k}+3\left(u_{k}\right)^{2} z_{k}+6 \partial_{t} u_{k} u_{k}\left(T_{\bar{M}}\left(v_{k}\right)-T_{\bar{M}}\left(Y_{k}\right)\right) \\
& +3 \partial_{t} u_{k}\left(T_{\bar{M}}\left(v_{k}\right)^{2}-T_{\bar{M}}\left(Y_{k}\right)^{2}\right)+6 u_{k}\left(T_{\bar{M}}\left(v_{k}\right) w_{k}-T_{\bar{M}}\left(Y_{k}\right) Z_{k}\right) \\
& +3\left(T_{\bar{M}}\left(v_{k}\right)^{2} w_{k}-T_{\bar{M}}\left(Y_{k}\right)^{2} Z_{k}\right) \\
= & \partial_{t} z_{k}-\Delta z_{k}+3\left(u_{k}\right)^{2} z_{k}+6 \partial_{t} u_{k} u_{k}\left(T_{\bar{M}}\left(v_{k}\right)-T_{\bar{M}}\left(Y_{k}\right)\right) \\
& +3 \partial_{t} u_{k}\left(T_{\bar{M}}\left(v_{k}\right)-T_{\bar{M}}\left(Y_{k}\right)\right)\left(T_{\bar{M}}\left(v_{k}\right)+T_{\bar{M}}\left(Y_{k}\right)\right)+6 u_{k} z_{k} T_{\bar{M}}\left(Y_{k}\right) \\
& +6 u_{k}\left(T_{\bar{M}}\left(v_{k}\right)-T_{\bar{M}}\left(Y_{k}\right)\right) w_{k}+3 z_{k} T_{\bar{M}}\left(Y_{k}\right)^{2} \\
& +3\left(T_{\bar{M}}\left(v_{k}\right)+T_{\bar{M}}\left(Y_{k}\right)\right)\left(T_{\bar{M}}\left(v_{k}\right)-T_{\bar{M}}\left(Y_{k}\right)\right) w_{k} \\
= & \partial_{t} z_{k}-\Delta z_{k}+R_{1, k} .
\end{aligned}
$$

Using (3.16), (3.18) and (3.21), we can estimate $R_{1, k}$

$$
\left|R_{1, k}\right| \leq M\left|z_{k}\right|+M\left|\bar{y}_{k}\right|\left(\left|\partial_{t} u_{k}\right|+\left|w_{k}\right|\right) .
$$

Moreover, from (3.34), we can write $D P_{k}\left(Z_{k}\right)\left(z_{k}\right)=\partial_{t} z_{k}-\Delta z_{k}+R_{2, k}$ where, according to (3.16) and (3.21)

$$
\left|R_{2, k}\right| \leq M\left|z_{k}\right|+M\left|T_{\bar{M}}^{\prime}\left(Y_{k}\right)\right|\left|\bar{y}_{k}\right|\left(\left|\partial_{t} u_{k}\right|+\left|Z_{k}\right|\right)
$$

We deduce from these inequalities that

$$
\begin{aligned}
& \int_{0}^{T} \int_{\Omega} e^{2 s \varphi}\left(P_{k} w_{k}-P_{k} Z_{k}\right) D P_{k}\left(Z_{k}\right)\left(z_{k}\right) \mathrm{d} x \mathrm{~d} t \geq \frac{3}{4} \int_{0}^{T} \int_{\Omega} e^{2 s \varphi}\left|\partial_{t} z_{k}-\Delta z_{k}\right|^{2} \mathrm{~d} x \mathrm{~d} t \\
& -M \int_{0}^{T} \int_{\Omega} e^{2 s \varphi}\left|z_{k}\right|^{2} \mathrm{~d} x \mathrm{~d} t-M \int_{0}^{T} \int_{\Omega} e^{2 s \varphi}\left|\bar{y}_{k}\right|^{2}\left(\left|\partial_{t} u_{k}\right|^{2}+\left|w_{k}\right|^{2}+\left|T_{\bar{M}}^{\prime}\left(Y_{k}\right)\right|^{2}\left|\partial_{t} u_{k}\right|^{2}\right) \mathrm{d} x \mathrm{~d} t \\
& -M \int_{0}^{T} \int_{\Omega} e^{2 s \varphi}\left|\bar{y}_{k}\right|^{2}\left|T_{\bar{M}}^{\prime}\left(Y_{k}\right)\right|^{2}\left|Z_{k}\right|^{2} \mathrm{~d} x \mathrm{~d} t
\end{aligned}
$$

According to (3.17) and using that $Z_{k}=w_{k}-z_{k}$ and that $\chi_{[-2 \bar{M}, 2 \bar{M}]}\left(Y_{k}\right) \leq \chi_{[-3 \bar{M}, 3 \bar{M}]}\left(\bar{y}_{k}\right)$ thanks to (3.4), we get for the last two terms that

$$
\begin{aligned}
& \int_{0}^{T} \int_{\Omega} e^{2 s \varphi}\left|\bar{y}_{k}\right|^{2}\left(\left|\partial_{t} u_{k}\right|^{2}+\left|w_{k}\right|^{2}+\left|T_{\bar{M}}^{\prime}\left(Y_{k}\right)\right|^{2}\left|\partial_{t} u_{k}\right|^{2}+\left|T_{\bar{M}}^{\prime}\left(Y_{k}\right)\right|^{2}\left|Z_{k}\right|^{2}\right) \mathrm{d} x \mathrm{~d} t \\
& \leq C \int_{0}^{T} \int_{\Omega} e^{2 s \varphi}\left|\bar{y}_{k}\right|^{2}\left(\left|\partial_{t} u_{k}\right|^{2}+\left|w_{k}\right|^{2}+\chi_{[-3 \bar{M}, 3 \bar{M}]}\left(\bar{y}_{k}\right)\left|z_{k}\right|^{2}\right) \mathrm{d} x \mathrm{~d} t .
\end{aligned}
$$

Let us estimate this last integral. We first notice that

$$
\int_{0}^{T} \int_{\Omega} e^{2 s \varphi}\left|\bar{y}_{k}\right|^{2} \chi_{[-3 \bar{M}, 3 \bar{M}]}\left(\bar{y}_{k}\right)\left|z_{k}\right|^{2} \mathrm{~d} x \mathrm{~d} t \leq M \int_{0}^{T} \int_{\Omega} e^{2 s \varphi}\left|z_{k}\right|^{2} \mathrm{~d} x \mathrm{~d} t .
$$


Next, according to (3.21) and (3.4), we have

$$
\begin{aligned}
\int_{0}^{T} \int_{\Omega} e^{2 s \varphi}\left|\bar{y}_{k}\right|^{2}\left(\left|\partial_{t} u_{k}\right|^{2}+\left|w_{k}\right|^{2}\right) \mathrm{d} x \mathrm{~d} t & \leq\left\|e^{s \varphi} \bar{y}_{k}\right\|_{L^{\infty}\left(0, T ; L^{2}(\Omega)\right)}^{2}\left(\left\|\partial_{t} u_{k}\right\|_{L^{2}\left(0, T ; L^{\infty}(\Omega)\right)}^{2}+\left\|w_{k}\right\|_{L^{2}\left(0, T ; L^{\infty}(\Omega)\right)}^{2}\right) \\
& \leq M\left\|e^{s \varphi} \bar{y}_{k}\right\|_{L^{\infty}\left(0, T ; L^{2}(\Omega)\right)}^{2} .
\end{aligned}
$$

We have, for all $t \in(0, T)$

$$
\begin{aligned}
\int_{\Omega} e^{2 s \varphi(t, x)}\left|\bar{y}_{k}(t, x)\right|^{2} \mathrm{~d} x & =\int_{\Omega} e^{2 s \varphi(t, x)}\left|\int_{T_{0}}^{t} z_{k}\left(t^{\prime}, x\right) \mathrm{d} t^{\prime}\right|^{2} \mathrm{~d} x \\
& \leq\left.\left. C \int_{\Omega} e^{2 s \varphi(t, x)}\left|\int_{T_{0}}^{t}\right| z_{k}\left(t^{\prime}, x\right)\right|^{2} d t^{\prime}\left|\mathrm{d} x \leq C \int_{0}^{T} \int_{\Omega} e^{2 s \varphi}\right| z_{k}\right|^{2} \mathrm{~d} x \mathrm{~d} t^{\prime}
\end{aligned}
$$

using inequality (C.5) for $\varphi$. By this way, we deduce that

$$
\int_{0}^{T} \int_{\Omega} e^{2 s \varphi}\left|\bar{y}_{k}\right|^{2}\left(\left|\partial_{t} u_{k}\right|^{2}+\left|w_{k}\right|^{2}\right) \mathrm{d} x \mathrm{~d} t \leq M \int_{0}^{T} \int_{\Omega} e^{2 s \varphi}\left|z_{k}\right|^{2} \mathrm{~d} x \mathrm{~d} t
$$

Using, (3.40), (3.41) and this last inequality, (3.39) becomes

$$
\begin{aligned}
\int_{0}^{T} \int_{\Omega} e^{2 s \varphi}\left(P_{k} w_{k}-P_{k} Z_{k}\right) D P_{k}\left(Z_{k}\right)\left(z_{k}\right) \mathrm{d} x \mathrm{~d} t & \geq \frac{3}{4} \int_{0}^{T} \int_{\Omega} e^{2 s \varphi}\left|\partial_{t} z_{k}-\Delta z_{k}\right|^{2} \mathrm{~d} x \mathrm{~d} t \\
& -M \int_{0}^{T} \int_{\Omega} e^{2 s \varphi}\left|z_{k}\right|^{2} \mathrm{~d} x \mathrm{~d} t
\end{aligned}
$$

- Step 2. To bound the second term in the right-hand side of (3.38), by definition (3.34) of $D P_{k}$, we have, according to (3.17) and (3.21)

$$
\left|D P_{k}\left(w_{k}\right)\left(z_{k}\right)\right|^{2} \leq 2\left|\partial_{t} z_{k}-\Delta z_{k}\right|^{2}+M\left|z_{k}\right|^{2}+M\left|\bar{y}_{k}\right|^{2}\left(\left|\partial_{t} u_{k}\right|^{2}+\left|w_{k}\right|^{2}\right) .
$$

Thus, using again (3.43), we get

$$
\frac{1}{4} \int_{0}^{T} \int_{\Omega} e^{2 s \varphi}\left|D P_{k}\left(w_{k}\right)\left(z_{k}\right)\right|^{2} \mathrm{~d} x \mathrm{~d} t \leq \frac{1}{2} \int_{0}^{T} \int_{\Omega} e^{2 s \varphi}\left|\partial_{t} z_{k}-\Delta z_{k}\right|^{2} \mathrm{~d} x \mathrm{~d} t+M \int_{0}^{T} \int_{\Omega} e^{2 s \varphi}\left|z_{k}\right|^{2} \mathrm{~d} x \mathrm{~d} t .
$$

- Step 3. To bound the last term of (3.38), we notice that

$$
\begin{aligned}
& D P_{k}\left(w_{k}\right)\left(z_{k}\right)-D P_{k}\left(Z_{k}\right)\left(z_{k}\right)=3 z_{k}\left(2 u_{k} T_{\bar{M}}\left(v_{k}\right)+T_{\bar{M}}\left(v_{k}\right)^{2}\right)-3 z_{k}\left(2 u_{k} T_{\bar{M}}\left(Y_{k}\right)+T_{\bar{M}}\left(Y_{k}\right)^{2}\right) \\
& +6 T_{\bar{M}}^{\prime}\left(v_{k}\right) \bar{y}_{k}\left(\partial_{t} u_{k} u_{k}+\partial_{t} u_{k} T_{\bar{M}}\left(v_{k}\right)+u_{k} w_{k}+w_{k} T_{\bar{M}}\left(v_{k}\right)\right) \\
& -6 T_{\bar{M}}^{\prime}\left(Y_{k}\right) \bar{y}_{k}\left(\partial_{t} u_{k} u_{k}+\partial_{t} u_{k} T_{\bar{M}}\left(Y_{k}\right)+u_{k} Z_{k}+Z_{k} T_{\bar{M}}\left(Y_{k}\right)\right) \\
& =6 z_{k} u_{k}\left(T_{\bar{M}}\left(v_{k}\right)-T_{\bar{M}}\left(Y_{k}\right)\right)+3 z_{k}\left(T_{\bar{M}}\left(v_{k}\right)^{2}-T_{\bar{M}}\left(Y_{k}\right)^{2}\right)+6 \partial_{t} u_{k} u_{k} \bar{y}_{k}\left(T_{\bar{M}}^{\prime}\left(v_{k}\right)-T_{\bar{M}}^{\prime}\left(Y_{k}\right)\right) \\
& +6 \partial_{t} u_{k} \bar{y}_{k}\left(T_{\bar{M}}^{\prime}\left(v_{k}\right)-T_{\bar{M}}^{\prime}\left(Y_{k}\right)\right) T_{\bar{M}}\left(v_{k}\right)+6 \partial_{t} u_{k} T_{\bar{M}}^{\prime}\left(Y_{k}\right) \bar{y}_{k}\left(T_{\bar{M}}\left(v_{k}\right)-T_{\bar{M}}\left(Y_{k}\right)\right) \\
& +6 u_{k} \bar{y}_{k}\left(T_{\bar{M}}^{\prime}\left(v_{k}\right)-T_{\bar{M}}^{\prime}\left(Y_{k}\right)\right) w_{k}+6 u_{k} T_{\bar{M}}^{\prime}\left(Y_{k}\right) \bar{y}_{k} z_{k}+6 \bar{y}_{k}\left(T_{\bar{M}}^{\prime}\left(v_{k}\right)-T_{\bar{M}}^{\prime}\left(Y_{k}\right)\right) w_{k} T_{\bar{M}}\left(v_{k}\right) \\
& +6 T_{\bar{M}}^{\prime}\left(Y_{k}\right) \bar{y}_{k} w_{k}\left(T_{\bar{M}}\left(v_{k}\right)-T_{\bar{M}}\left(Y_{k}\right)\right)+6 T_{\bar{M}}^{\prime}\left(Y_{k}\right) \bar{y}_{k} z_{k} T_{\bar{M}}\left(Y_{k}\right) .
\end{aligned}
$$


Hence, using that $\left|T_{\bar{M}}^{\prime}\left(Y_{k}\right)\right| \leq L \chi_{[-2 \bar{M}, 2 \bar{M}]}\left(Y_{k}\right) \leq L \chi_{[-3 \bar{M}, 3 \bar{M}]}\left(\bar{y}_{k}\right)$

$$
\left|D P_{k}\left(w_{k}\right)\left(z_{k}\right)-D P_{k}\left(Z_{k}\right)\left(z_{k}\right)\right| \leq M\left|z_{k}\right|+M\left|\bar{y}_{k}\right|\left(\left|\partial_{t} u_{k}\right|+\left|w_{k}\right|+\left|z_{k}\right| \chi_{[-\overline{3 M}, \overline{3 M}]}\left(\bar{y}_{k}\right)\right) .
$$

This implies that

$$
\begin{aligned}
& \int_{0}^{T} \int_{\Omega} e^{2 s \varphi}\left|P_{k} w_{k}\right|\left|D P_{k}\left(w_{k}\right)\left(z_{k}\right)-D P_{k}\left(Z_{k}\right)\left(z_{k}\right)\right| \mathrm{d} x \mathrm{~d} t \\
& \leq \frac{1}{2} \int_{0}^{T} \int_{\Omega} e^{2 s \varphi}\left|P_{k} w_{k}\right|^{2} \mathrm{~d} x \mathrm{~d} t+\frac{1}{2} \int_{0}^{T} \int_{\Omega} e^{2 s \varphi}\left|D P_{k}\left(w_{k}\right)\left(z_{k}\right)-D P_{k}\left(Z_{k}\right)\left(z_{k}\right)\right|^{2} \mathrm{~d} x \mathrm{~d} t \\
& \leq \frac{1}{2} \int_{0}^{T} \int_{\Omega} e^{2 s \varphi}\left|f_{k}\right|^{2} \mathrm{~d} x \mathrm{~d} t+M \int_{0}^{T} \int_{\Omega} e^{2 s \varphi}\left|z_{k}\right|^{2} \mathrm{~d} x \mathrm{~d} t
\end{aligned}
$$

according to (3.41) and (3.43).

Using (3.44), (3.45) and (3.46), inequality (3.38) becomes:

$$
\begin{aligned}
\frac{1}{4} \int_{0}^{T} \int_{\Omega} e^{2 s \varphi} \mid & \partial_{t} z_{k}-\left.\Delta z_{k}\right|^{2} \mathrm{~d} x \mathrm{~d} t+s \int_{0}^{T} \int_{\Gamma} e^{2 s \varphi} \theta\left|\nabla z_{k} \cdot n\right|^{2} \mathrm{~d} \gamma \mathrm{d} t \\
\leq & \frac{3}{2} \int_{0}^{T} \int_{\Omega} e^{2 s \varphi}\left|f_{k}\right|^{2} \mathrm{~d} x \mathrm{~d} t+M \int_{0}^{T} \int_{\Omega} e^{2 s \varphi}\left|z_{k}\right|^{2} \mathrm{~d} x \mathrm{~d} t .
\end{aligned}
$$

Using Theorem 2.1, we can eliminate the last term in the right hand-side of (3.47) for $s$ larger than some constant $s_{0}$. Thus, using inequality (2.7), we get the following bound on $z_{k}\left(T_{0}\right)$ :

$$
s \int_{\Omega} e^{2 s \varphi\left(T_{0}\right)}\left|z_{k}\left(T_{0}\right)\right|^{2} \mathrm{~d} x \leq M \int_{0}^{T} \int_{\Omega} e^{2 s \varphi}\left|f_{k}\right|^{2} \mathrm{~d} x \mathrm{~d} t .
$$

In the left hand-side of this inequality, we have $z_{k}\left(T_{0}, x\right)=w_{k}\left(T_{0}, x\right)-Z_{k}\left(T_{0}, x\right)$ for $x \in \Omega$ and, using (3.8) and (3.14), we get that

$$
z_{k}\left(T_{0}, x\right)=-h\left(T_{0}, x\right)\left(\widetilde{\sigma}_{k+1}(x)-\sigma(x)\right), \quad x \in \Omega .
$$

In the right hand-side of (3.48), since $f_{k}=\left(\sigma_{k}-\sigma\right) \partial_{t} h$ and $h$ is assumed to be bounded in $H^{1}\left(0, T ; L^{\infty}(\Omega)\right)$, we have

$$
\int_{0}^{T} \int_{\Omega} e^{2 s \varphi}\left|f_{k}\right|^{2} \mathrm{~d} x \mathrm{~d} t \leq M \int_{\Omega} e^{2 s \varphi\left(T_{0}\right)}\left|\sigma_{k}-\sigma\right|^{2} \mathrm{~d} x
$$

Using (3.2), we get that

$$
s \int_{\Omega} e^{2 s \varphi\left(T_{0}\right)}\left|\widetilde{\sigma}_{k+1}-\sigma\right|^{2} \mathrm{~d} x \leq M \int_{\Omega} e^{2 s \varphi\left(T_{0}\right)}\left|\sigma_{k}-\sigma\right|^{2} \mathrm{~d} x .
$$

Now, to estimate $\sigma_{k+1}=\Pi_{\sigma_{\max }}\left(\widetilde{\sigma}_{k+1}\right)$, we notice that, since $\sigma$ satisfies (1.3), we have

$$
\left|\sigma_{k+1}-\sigma\right| \leq\left|\widetilde{\sigma}_{k+1}-\sigma\right| \text { in } \Omega
$$


Thus, we get (3.15) and, applying iteratively this estimate, we obtain that

$$
\int_{\Omega} e^{2 s \varphi\left(T_{0}\right)}\left|\sigma_{k+1}-\sigma\right|^{2} \mathrm{~d} x \leq\left(\frac{M}{s}\right)^{k+1} \int_{\Omega} e^{2 s \varphi\left(T_{0}\right)}\left|\sigma_{0}-\sigma\right|^{2} \mathrm{~d} x
$$

Thus, for $s$ large enough we deduce from this inequality the convergence of the sequence $\left(\sigma_{k}\right)_{k \in \mathbb{N}}$ to $\sigma$.

This concludes the proof of Theorem 3.4.

\section{NUMERICAL ISSUES}

\subsection{Numerical methods}

In this subsection, we present the discretization procedure and the numerical methods used in our numerical simulations. To simplify the presentation, we explain the discretization scheme in the one-dimensional case and assume that $\Omega=(0, L)$ for $L>0$ and $\Gamma=\{x=L\}$.

\subsubsection{Generation of the data}

In this article, we work with synthetic data. To discretize the reaction-diffusion equation (1.1) for the exact source $\sigma$, we use a finite differences scheme based on the three-point backward Euler scheme and a linearization of the cubic term. We denote by $N_{x} \in \mathbb{N}$ the number of discretization points in the interior of $[0, L]$ and by $N_{t} \in \mathbb{N}$ the number of discretization points in the interior of $[0, T]$. The space and time steps are denoted by $\Delta x=\frac{L}{N_{x}+1}$ and $\Delta t=\frac{T}{N_{t}+1}$ respectively and we define, for $0 \leq j \leq N_{x}+1$ and $0 \leq n \leq N_{t}+1, u_{j}^{n}$ a numerical approximation of the solution $u\left(t^{n}, x_{j}\right)$ with $t^{n}=n \Delta t$ and $x_{j}=j \Delta x$. The approximated solution is computed in the following way:

Initialize: $u_{j}^{0}=u_{\circ}\left(x_{j}\right), \quad 0 \leq j \leq N_{x}+1$.

For $0 \leq n \leq N_{t}$, knowing $u^{n}$, compute $u^{n+1}$ as the solution of the linear system:

$$
\left\{\begin{array}{l}
\frac{u_{j}^{n+1}-u_{j}^{n}}{\Delta t}-\frac{u_{j+1}^{n+1}-2 u_{j}^{n+1}+u_{j-1}^{n+1}}{\Delta x^{2}}+\left(u_{j}^{n}\right)^{3}+3\left(u_{j}^{n}\right)^{2}\left(u_{j}^{n+1}-u_{j}^{n}\right)=\sigma\left(x_{j}\right) h\left(t^{n}, x_{j}\right), \\
u_{0}^{n+1}=g\left(t^{n+1}, 0\right) \quad \text { and } u_{N_{x}+1}^{n+1}=g\left(t^{n+1}, L\right), \quad 1 \leq j \leq N_{x},
\end{array}\right.
$$

where the time implicit cubic term $\left(u_{j}^{n+1}\right)^{3}$ has been approximated by its first order Taylor expansion $\left(u_{j}^{n}\right)^{3}+$ $3\left(u_{j}^{n}\right)^{2}\left(u_{j}^{n+1}-u_{j}^{n}\right)$. Then, we compute the counterpart of the continuous measurements $r$ and $m$ given in (1.2) as follows:

$$
m^{n}=\frac{u_{N_{x}+1}^{n}-u_{N_{x}}^{n}}{\Delta x}, \quad 0 \leq n \leq N_{t}+1 \quad \text { and } \quad r_{j}=u_{j}^{n_{0}}, \quad 0 \leq j \leq N_{x}+1
$$

with $n_{0}$ is the integer part of $N_{t} / 2+1$.

On the computed data, we may add a Gaussian noise:

$$
\begin{array}{cl}
m^{n} \longleftarrow m^{n}+\alpha\left(\max _{n} m^{n}\right) \mathcal{N}(0,1), & 0 \leq n \leq N_{t}+1, \\
r_{j} \longleftarrow r_{j}+\alpha\left(\max _{j} r_{j}\right) \mathcal{N}(0,1), & 0 \leq j \leq N_{x}+1,
\end{array}
$$

where $\mathcal{N}(0,1)$ satisfies a centered normal law with deviation 1 and $\alpha$ is the level of noise (i.e. $\alpha=0.01$ corresponds to a noise of $1 \%)$. 


\subsubsection{Discrete algorithm}

We present in this subsection the discrete version of Algorithm 3.2. In order to lighten the notations, we will denote by $\bar{\sigma}$ the source term at the current iteration (previously denoted by $\sigma_{k}$ in the continuous framework).

Algorithm 4.1. Initialisation: Start with $\bar{\sigma}=0$.

Iteration : Until the convergence criteria is reached, do

- Step 1 - Knowing $\bar{\sigma} \in \mathbb{R}^{N_{x}}$, solve

$$
\left\{\begin{array}{l}
\frac{\bar{u}_{j}^{n+1}-\bar{u}_{j}^{n}}{\Delta t}-\frac{\bar{u}_{j+1}^{n+1}-2 \bar{u}_{j}^{n+1}+\bar{u}_{j-1}^{n+1}}{\Delta x^{2}}+\left(\bar{u}_{j}^{n}\right)^{3}=\bar{\sigma}_{j} h\left(t^{n}, x_{j}\right), \\
\bar{u}_{0}^{n+1}=g\left(t^{n+1}, 0\right) \quad \text { and } \bar{u}_{N_{x}+1}^{n+1}=g\left(t^{n+1}, L\right), \quad 0 \leq n \leq N_{t}, \\
\bar{u}_{j}^{0}=u_{\circ}\left(x_{j}\right), \quad 1 \leq j \leq N_{x},
\end{array}\right.
$$

and set $v_{j}=r_{j}-\bar{u}_{j}^{n_{0}}$.

- Step 2 - Define for $1 \leq n \leq N_{t}$,

$$
\mu^{n}=\frac{\left(m-\frac{\bar{u}_{N_{x}+1}-\bar{u}_{N_{x}}}{\Delta x}\right)^{n+1}-\left(m-\frac{\bar{u}_{N_{x}+1}-\bar{u}_{N_{x}}}{\Delta x}\right)^{n-1}}{2 \Delta t}
$$

and discretize the functional (3.11) as follows:

$$
J_{0, k}[\mu](z)=\frac{1}{2} \Delta t \Delta x \sum_{n=1}^{N_{t}} \sum_{j=1}^{N_{x}} e^{2 s \varphi\left(t^{n}, x_{j}\right)}\left|\left(P_{k} z\right)_{j}^{n}\right|^{2}+\frac{s}{2} \Delta t \sum_{n=1}^{N_{t}} e^{2 s \varphi\left(t^{n}, L\right)} \theta\left(t^{n}\right)\left|\frac{-z_{N_{x}}^{n}}{\Delta x}-\mu^{n}\right|^{2},
$$

where

$$
\begin{aligned}
\left(P_{k} z\right)_{j}^{n} & =\frac{z_{j}^{n+1}-z_{j}^{n-1}}{2 \Delta t}-\frac{z_{j+1}^{n}-2 z_{j}^{n}+z_{j-1}^{n}}{\Delta x^{2}}+3\left(\bar{u}_{j}^{n}\right)^{2} z_{j}^{n}+6 \frac{\bar{u}_{j}^{n+1}-\bar{u}_{j}^{n-1}}{2 \Delta t} \bar{u}_{j}^{n} T_{\bar{M}}\left(y_{j}^{n}\right) \\
& +3 \frac{\bar{u}_{j}^{n+1}-\bar{u}_{j}^{n-1}}{2 \Delta t} T_{\bar{M}}\left(y_{j}^{n}\right)^{2}+6 \bar{u}_{j}^{n} z_{j}^{n} T_{\bar{M}}\left(y_{j}^{n}\right)+3 z_{j}^{n} T_{\bar{M}}\left(y_{j}^{n}\right)^{2},
\end{aligned}
$$

with $z_{0}^{n}=z_{N_{x}+1}^{n}=0$ for all $0 \leq n \leq N_{t}+1$ and

$$
\begin{cases}y_{j}^{n_{0}}=v_{j}, \quad 1 \leq j \leq N_{x}, \\ y_{j}^{n}=y_{j}^{n-1}+\Delta t z_{j}^{n}, \quad \text { if } n>n_{0}, \\ y_{j}^{n}=y_{j}^{n+1}-\Delta t z_{j}^{n}, \quad \text { if } n<n_{0} .\end{cases}
$$

Find a critical point of $J_{0, k}[\mu]$ which is denoted by $Z=\left(Z_{j}^{n}\right)_{1 \leq j \leq N_{x}, 0 \leq n \leq N_{t}+1}$

- Step 3 - Update

$$
\bar{\sigma}_{j} \longleftarrow \bar{\sigma}_{j}+\frac{Z_{j}^{n_{0}}-\frac{v_{j+1}-2 v_{j}+v_{j-1}}{\Delta x^{2}}+v_{j} q\left[v_{j}, \bar{u}_{j}^{n_{0}}\right]}{h\left(t^{n_{0}}, x_{j}\right)}, \quad 1 \leq j \leq N_{x} .
$$

- Step 4 - At last, define

$$
\bar{\sigma}_{j} \longleftarrow \operatorname{sign}\left(\bar{\sigma}_{j}\right) \min \left(\sigma_{\max },\left|\bar{\sigma}_{j}\right|\right) .
$$


The iterative loop is stopped when two consecutive $\bar{\sigma}$ are closer than a fixed relative tolerance $\varepsilon$ or when the maximal number of iterations is reached. In the absence of knowledge of the exact solution $\sigma$, the quality of the converged solution is measured thanks to the following criteria

$$
\operatorname{err}_{r}=\frac{\left\|r-\bar{u}^{n_{0}}\right\|_{2}}{\|r\|_{2}} \quad \text { and } \quad \operatorname{err}_{m}=\frac{\left\|m-\frac{\bar{u}_{N_{x}+1}-\bar{u}_{N_{x}}}{\Delta x}\right\|_{2}}{\|m\|_{2}}
$$

that should be of the order of the noise level on the observations. If the exact solution $\sigma$ is known, we can also compute the relative error

$$
\operatorname{err}_{\sigma}=\frac{\|\sigma-\bar{\sigma}\|_{2}}{\|\sigma\|_{2}}
$$

Remark 4.2. For the numerical implementation of Step 2 in Algorithm 4.1, we determine a critical point of $J_{0, k}[\mu]$ by applying the Newton method or Newton-Krylov method $[4,21]$. This last method belongs to the family of inexact Newton methods and consists of solving at each step the linear system in a Krylov subspace. The Newton minimization method is globally convergent if the functional is strongly convex. To be in this framework, it would be necessary to prove that the discrete functional $J_{0, k}(4.5)$ is strongly convex under boundedness assumptions (like in Lemma 3.8 for the functional in the continuous setting) and to prove that the discrete minimization sequence satisfies these bounds. A complete study of the discretized algorithm could be tackled in the future and would involve in particular a Carleman inequality for the discretized heat equation. In our numerical simulations, we have taken the initial guess of the iterative Newton-Krylov method equal to 0 and checked that the convergence does not depend on this initialization. Thus, we observe numerically global convergence properties for the minimization of $J_{0, k}[\mu]$.

Remark 4.3. In order to avoid the inverse crime, we introduce a bias by taking different schemes for the direct and the inverse problems. Hence, we solve (4.1) associated to $\sigma$ thanks to a linearized implicit scheme and we use an explicit scheme for the nonlinear term in equation (4.3) with $\bar{\sigma}=\sigma_{k}$.

\subsubsection{Numerical challenges}

One of the main drawbacks of the numerical method presented in Algorithm 3.2 is that we have to differentiate in time the observation $m$ in (4.4) and to take the Laplacian of the observation $r$ in (4.7). Thus, even a small perturbation (noise) on the observations may induce a large perturbation on its derivatives. In order to partially remedy this problem in the presence of noise, we first regularize the data $(m, r)$ thanks to a 3-order low-pass Butterworth filter [6] associated to a cutoff frequency $\omega$. We also replace the classical finite difference formulae in (4.4) and (4.7) that generate instabilities by a Savitzki-Golay formula [25] associated with a cubic polynomial and a window size of 5 points.

As already mentioned previously, another difficulty is the presence of the exponential weights in the functional that leads to severe numerical difficulties when performing the minimization for $s$ large. Indeed, to ensure the strong convexity of the functional $J_{0, k}$ (see Lem. 3.8) and the convergence of Algorithm 3.2 (see Thm. 3.4), s has to be large. In [2], this difficulty was solved by choosing a functional that only depended on the conjugate variable $e^{s \varphi} z$ and the corresponding conjugate operator. But this was possible because the considered operator was linear. Here, we managed to deal with this difficulty by introducing the new weight functions (2.4). In Figure 1, we plot $e^{s \varphi}$ in $(0, T) \times(0, L)$ for $s=1$ and $s=100$. Notice that even for $s$ large, the function does not vanish at the observation time $T_{0}=0.5$ what allows a good reconstruction of the source term in the whole domain $\Omega$. Numerically, we observe that for $s=1$, the minimisation step is slow (5202 seconds for $s=1$ versus 17 seconds for $s=100$ for the test case of Fig. 3a) and in some cases the convergence of the algorithm is not achieved (for example in the test case of Fig. $3 \mathrm{~b}$ ). 


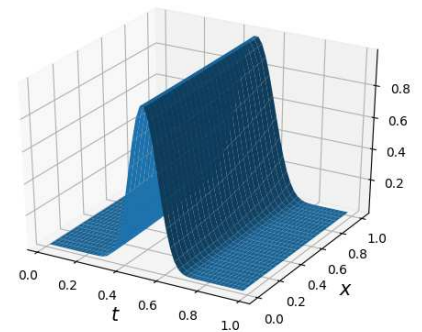

(a) $s=1$

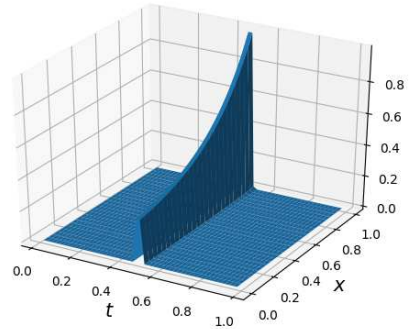

(b) $s=100$

FiguRE 1. Carleman weight function $e^{s \varphi}$ defined in (2.4) for different values of $s$.

TABLE 1. Numerical values for the variables.

\begin{tabular}{lcccccc}
\hline$L$ & $T$ & $N_{x}$ & $N_{t}$ & $g$ & $u_{\circ}$ & $\sigma_{\max }$ \\
\hline 1 & 1 & 25 & 50 & 0 & 0 & 2 \\
$\alpha$ & $x_{0}$ & $s$ & $\bar{M}$ & $\rho$ & $\varepsilon$ & $\omega$ \\
0 & -0.3 & 100 & 10 & $10^{-3}$ & $10^{-3}$ & 0.15 \\
\hline
\end{tabular}

\subsection{Numerical results}

This subsection is devoted to the presentation of some numerical examples to illustrate the properties of the numerical reconstruction method and its efficiency. All simulations are executed with PyTHON. The source codes are available on request. Table 1 gathers the numerical values used for all the following examples, unless specified otherwise where appropriate. Moreover, we construct the function $\Phi$ introduced in (3.10) in the form:

$$
\Phi(X)=\left\{\begin{array}{lr}
1, & \text { if }|X| \leq 1, \\
1-\frac{\int_{1}^{|X|} \exp \left(\frac{-1}{(x-1)(2-x)}\right) \mathrm{d} x}{\int_{1}^{2} \exp \left(\frac{-1}{(x-1)(2-x)}\right) \mathrm{d} x}, & \text { if } 1<|X|<2, \\
0, & \text { if }|X| \geq 2 .
\end{array}\right.
$$

Figure 2 presents some examples of data generated by the direct problem. In all the figures presenting the numerical results, the exact source that we want to recover is plotted by a red line, whereas the numerical source recovered by our method is represented by a dotted black line. The convergence informations (number of iterations, running time, convergence errors) are reported in Table 2.

\subsubsection{Simulations from data without noise}

In Figure 3, we present the successive results obtained at each iteration of Algorithm 3.2 in the case of the reconstruction of the source $\sigma(x)=\sin (\pi x)$ for two different choices of $h$. One can observe that in both cases the convergence criteria (4.8) for $\varepsilon$ is achieved in less than 20 iterations. In Figure 4, several results of reconstruction of sources obtained using Algorithm 3.2 in the absence of noise are given. 


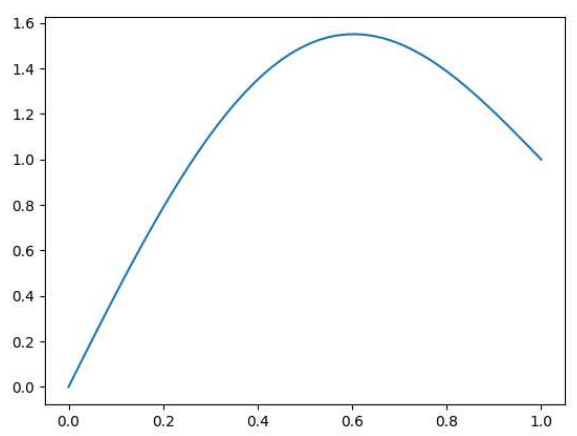

(a) $h(t)=t+\sin (\pi t)$

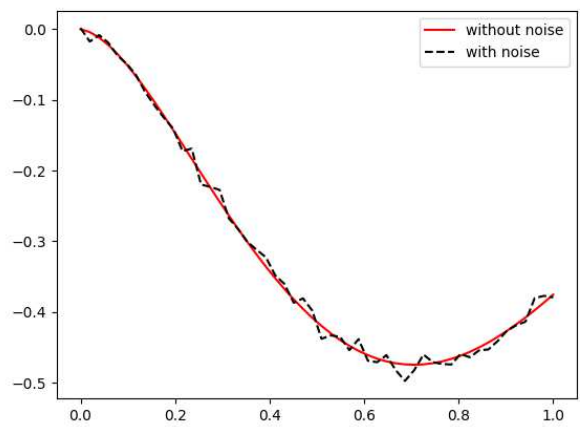

(c) $m(t)$ the measurement of the flux at $x=L$

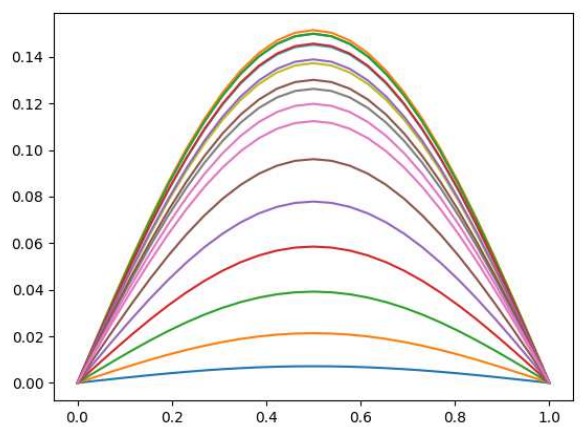

(b) $u(t, \cdot)$ for different times

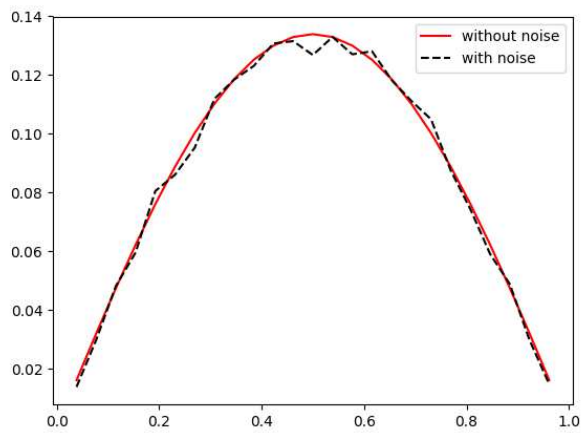

(d) $r(x)$ the measurement of $u$ at $t=T_{0}$

Figure 2. Examples of data used in the numerical examples for $\sigma(x)=\sin (\pi x)$ and $\alpha=2 \%$.

TABLE 2. Convergence results of the test cases.

\begin{tabular}{lccccc}
\hline Example & Number of iterations & Running time in seconds & $\operatorname{err}_{m}$ & $\operatorname{err}_{r}$ & $\operatorname{err}_{\sigma}$ \\
\hline Figure 3a & 3 & 117 & $0.1 \%$ & $0.2 \%$ & $0.02 \%$ \\
Figure 3b & 16 & 554 & $0.7 \%$ & $0.1 \%$ & $0.8 \%$ \\
Figure 5a & 3 & 87 & $1 \%$ & $0.3 \%$ & $2 \%$ \\
Figure 5b & 3 & 91 & $1 \%$ & $0.3 \%$ & $4 \%$ \\
Figure 5c & 3 & 97 & $3 \%$ & $0.5 \%$ & $9 \%$ \\
Figure 6b & 4 & 497 & $0.05 \%$ & $0.1 \%$ & $0.05 \%$ \\
Figure 6d & 6 & 802 & $0.1 \%$ & $0.1 \%$ & $0.01 \%$ \\
\hline
\end{tabular}

\subsubsection{Simulations with several levels of noise}

Figure 5 shows the results for $\sigma(x)=\sin (\pi x)$ with different levels of noise in the measurements $(\alpha=1 \%, 2 \%$ and $5 \%$ ). In Table 2, we report the corresponding errors on the reconstructed source. In fact, we observe that a noise of level $\alpha$ in the measurements gives rise to an error of order $2 \alpha$ in the recovered source.

\subsubsection{Simulations in two dimensions}

We also performed some reconstructions in two dimensions where $\Omega=(0,1)^{2}, x_{0}=(-0.3,-0.3)$ and $\Gamma=$ $(\{0\} \times[0,1]) \cup([0,1] \times\{0\})$. By this way, assumption (2.5) is satisfied. Figure 6 presents the results obtained 


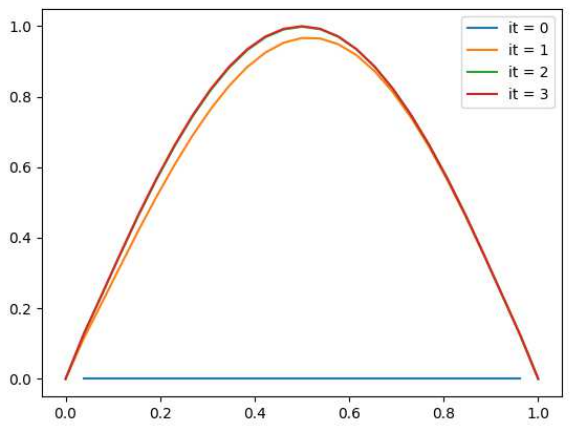

(a) $h(t)=t+\sin (\pi t)$

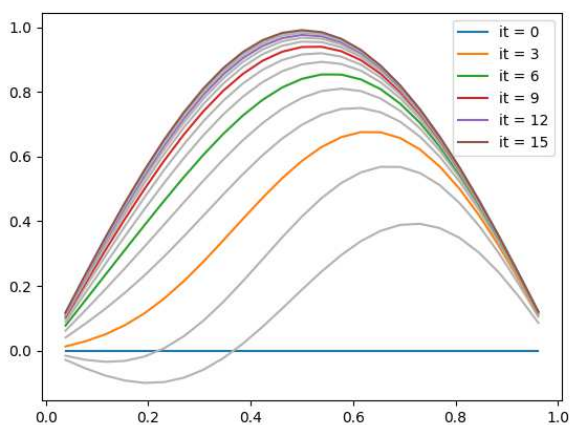

(b) $h(t)=t+\sin (7 \pi t)$

Figure 3. Reconstruction of $\sigma(x)=\sin (\pi x)$. Different choices for $h$ and the corresponding convergence history.

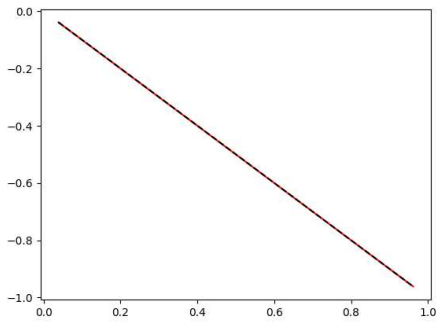

(a) $\sigma(x)=-x$

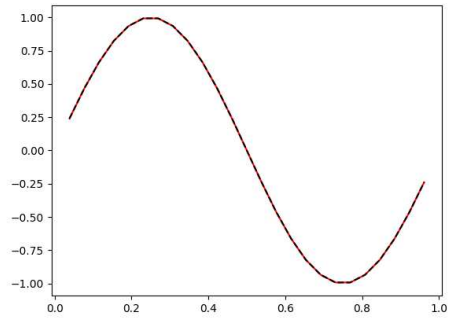

(b) $\sigma(x)=\sin (2 \pi x)$

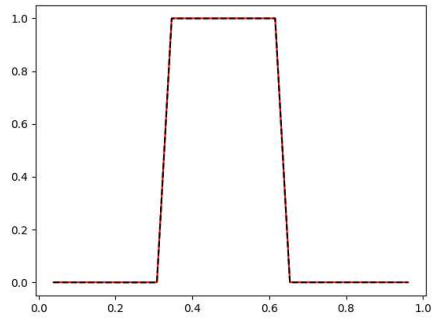

(c) $\sigma$ rectangular

FiguRE 4. Different examples of reconstruction for $h(t)=t+\sin (\pi t)$.

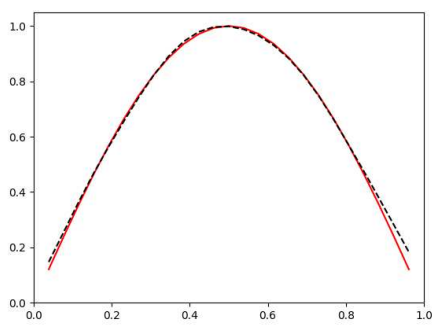

(a) $\alpha=1 \%$

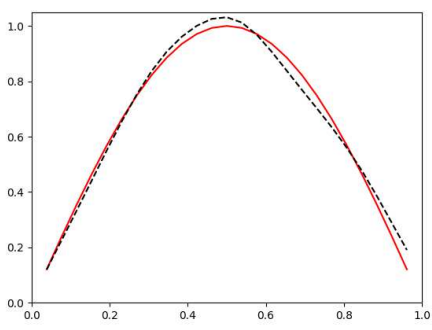

(b) $\alpha=2 \%$

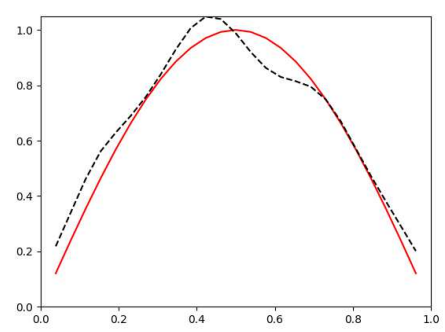

(c) $\alpha=5 \%$

FiguRE 5. Reconstruction of the source $\sigma(x)=\sin (\pi x)$ for $h(t)=t+\sin (\pi t)$ in presence of noise in the data. The level of noise is denoted by $\alpha$.

for two different sources in the absence of additional noise. The gray scales are identical for the exact and the recovered graphics. The final error (reported in Tab. 2) is less than $0.1 \%$ what shows the effectiveness of the reconstruction obtained in a few minutes on a personal laptop. 

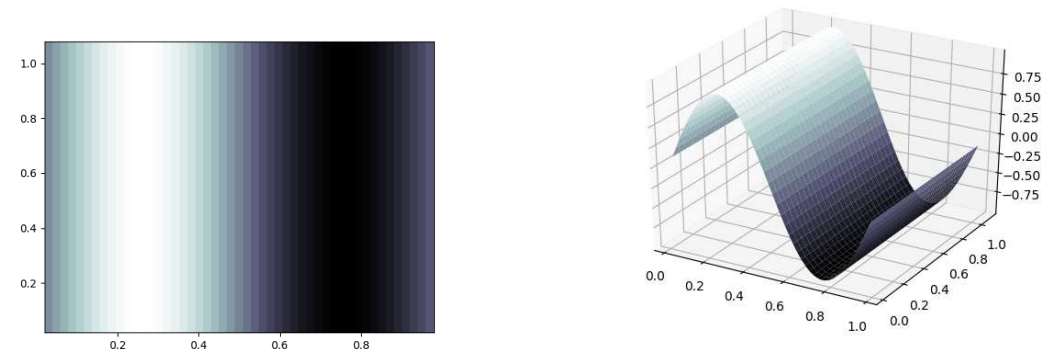

(a) Exact sources.
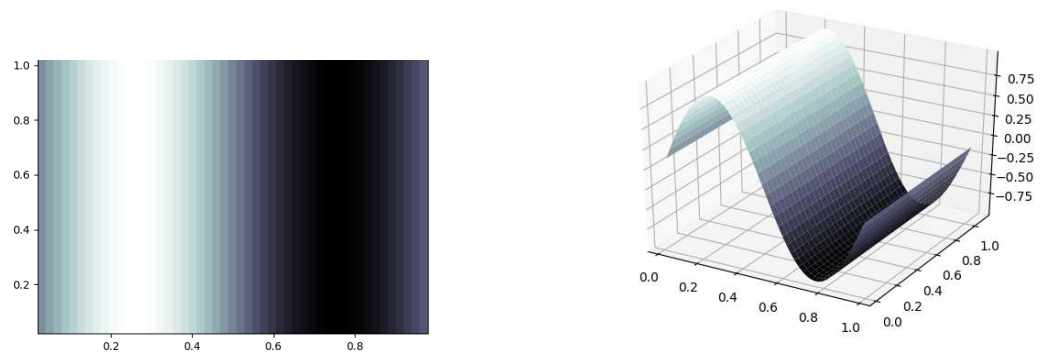

(b) Sources recovered numerically.
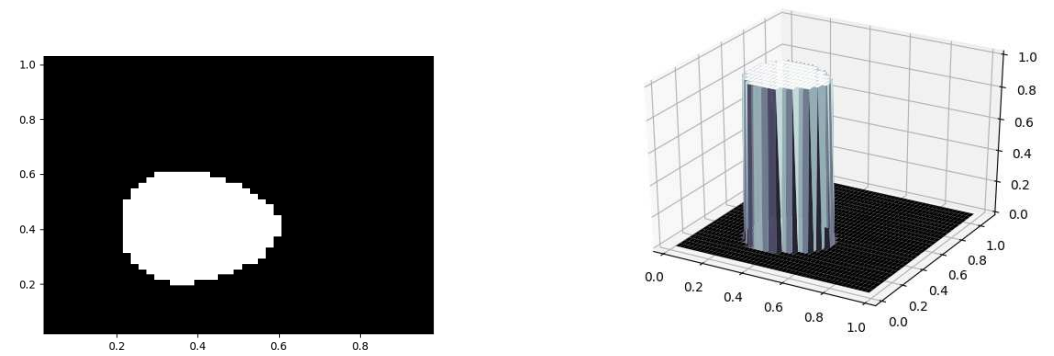

(c) Exact sources.
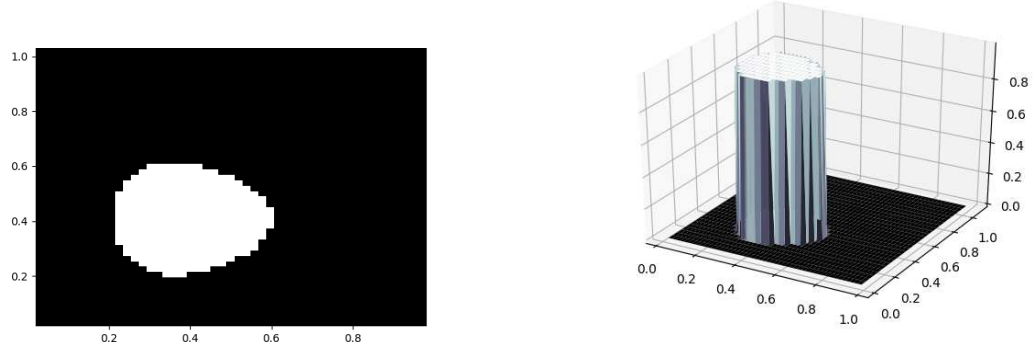

(d) Sources recovered numerically.

FIGURE 6. Different examples of reconstruction in the $2 \mathrm{~d}$ case. 


\section{Appendix A. Proof of the Carleman inequality given By THEOREM 2.1}

If we are in Case 1 with the first choice of weight (2.1), this result is proved in an identical way as Lemma 1.2 in [13] which considers the case of internal measurements. Assume now that we are in Case $\mathbf{2}$ where $\theta$ and $\psi$ are given by (2.4).

Let us give some properties on $\varphi$ which will be useful in what follows:

$$
\begin{gathered}
\varphi(t, x) \leq \varphi\left(T_{0}, x\right), \forall(t, x) \in(0, T) \times \Omega, \quad \nabla^{2} \varphi=2 \theta I_{d}, \\
|\nabla \varphi| \leq C \theta, \quad\left|\partial_{t} \varphi\right| \leq C \theta^{2}, \quad\left|\partial_{t} \nabla \varphi\right| \leq C \theta^{2}, \quad\left|\partial_{t t} \varphi\right| \leq C \theta^{3} .
\end{gathered}
$$

In the proof, we assume that $z$ belongs to $C^{2}([0, T] \times \bar{\Omega})$ and satisfies $z=0$ on $(0, T) \times \partial \Omega$. A density argument allows to come back to the regularity hypotheses of the theorem.

For all $s>0$, we set $w=e^{s \varphi} z$ and we introduce the conjugate operator $Q$ defined by

$$
Q w=e^{s \varphi}\left(\partial_{t}-\Delta\right)\left(e^{-s \varphi} w\right)
$$

If we set $f=\partial_{t} z-\Delta z$, we have

$$
Q w=e^{s \varphi} f
$$

Some computations give

$$
Q w=\partial_{t} w+2 s \nabla \varphi \cdot \nabla w+s \Delta \varphi w-\Delta w-\left(s^{2}|\nabla \varphi|^{2}+s \partial_{t} \varphi\right) w=Q_{+} w+Q_{-} w
$$

where the operators $Q_{+}$and $Q_{-}$are defined by

$$
\begin{aligned}
& Q_{+} w=-\Delta w-\left(s^{2}|\nabla \varphi|^{2}+s \partial_{t} \varphi\right) w \\
& Q_{-} w=\partial_{t} w+2 s \nabla \varphi \cdot \nabla w+s \Delta \varphi w .
\end{aligned}
$$

In a classical way, we write that

$$
\int_{0}^{T} \int_{\Omega} e^{2 s \varphi}|f|^{2} \mathrm{~d} x \mathrm{~d} t=\int_{0}^{T} \int_{\Omega}\left|Q_{+} w\right|^{2} \mathrm{~d} x \mathrm{~d} t+\int_{0}^{T} \int_{\Omega}\left|Q_{-} w\right|^{2} \mathrm{~d} x \mathrm{~d} t+2 \int_{0}^{T} \int_{\Omega} Q_{+} w Q_{-} w \mathrm{~d} x \mathrm{~d} t .
$$

The main part of the proof consists of bounding from below the terms in the right hand side by positive and dominant terms and a negative observation term located in $(0, T) \times \Gamma$. For the sake of clarity, we divide the proof in several steps.

- Step 1 - Explicit calculation of the cross-term.

We set

$$
\int_{0}^{T} \int_{\Omega} Q_{+} w Q_{-} w \mathrm{~d} x \mathrm{~d} t=\sum_{1 \leq i \leq 2,1 \leq k \leq 3} I_{i, k}
$$

where $I_{i, k}$ is the integral of the product of the $i$ th-term in $Q_{+} w$ and the $k$ th-term in $Q_{-} w$. 
Integrations by parts in time give easily

$$
\begin{aligned}
I_{11} & =\int_{0}^{T} \int_{\Omega}(-\Delta w) \partial_{t} w \mathrm{~d} x \mathrm{~d} t=\int_{0}^{T} \int_{\Omega} \nabla w \cdot \nabla \partial_{t} w \mathrm{~d} x \mathrm{~d} t-\int_{0}^{T} \int_{\partial \Omega} \nabla w \cdot n \partial_{t} w \mathrm{~d} \gamma \mathrm{d} t \\
& =\frac{1}{2}\left[\int_{\Omega}|\nabla w|^{2} \mathrm{~d} x\right]_{0}^{T}-\int_{0}^{T} \int_{\partial \Omega} \nabla w \cdot n \partial_{t} w \mathrm{~d} \gamma \mathrm{d} t=0
\end{aligned}
$$

since $w(0)=w(T)=0$ in $\Omega$ and $w=0$ on $(0, T) \times \partial \Omega$. An integration by parts in time gives for $I_{21}$

$$
I_{21}=-\int_{0}^{T} \int_{\Omega}\left(s^{2}|\nabla \varphi|^{2}+s \partial_{t} \varphi\right) w \partial_{t} w \mathrm{~d} x \mathrm{~d} t=\frac{1}{2} \int_{0}^{T} \int_{\Omega} \partial_{t}\left(s^{2}|\nabla \varphi|^{2}+s \partial_{t} \varphi\right)|w|^{2} \mathrm{~d} x \mathrm{~d} t .
$$

We compute in the same way, by integrating by parts in space

$$
\begin{aligned}
I_{12}= & -\int_{0}^{T} \int_{\Omega} \Delta w(2 s \nabla \varphi \cdot \nabla w) \mathrm{d} x \mathrm{~d} t \\
= & 2 s \int_{0}^{T} \int_{\Omega} \nabla w \cdot \nabla(\nabla \varphi \cdot \nabla w) \mathrm{d} x \mathrm{~d} t-2 s \int_{0}^{T} \int_{\partial \Omega} \nabla w \cdot n(\nabla \varphi \cdot \nabla w) \mathrm{d} \gamma \mathrm{d} t \\
= & 2 s \int_{0}^{T} \int_{\Omega}\left(\nabla^{2} \varphi\right) \nabla w \cdot \nabla w \mathrm{~d} x \mathrm{~d} t+2 s \int_{0}^{T} \int_{\Omega}\left(\nabla^{2} w\right) \nabla w \cdot \nabla \varphi \mathrm{d} x \mathrm{~d} t-2 s \int_{0}^{T} \int_{\partial \Omega} \nabla w \cdot n(\nabla \varphi \cdot \nabla w) \mathrm{d} \gamma \mathrm{d} t \\
= & 2 s \int_{0}^{T} \int_{\Omega}\left(\nabla^{2} \varphi\right) \nabla w \cdot \nabla w \mathrm{~d} x \mathrm{~d} t-s \int_{0}^{T} \int_{\Omega}|\nabla w|^{2} \Delta \varphi \mathrm{d} x \mathrm{~d} t+s \int_{0}^{T} \int_{\partial \Omega}|\nabla w|^{2} \nabla \varphi \cdot n \mathrm{~d} \gamma \mathrm{d} t \\
& -2 s \int_{0}^{T} \int_{\partial \Omega} \nabla w \cdot n(\nabla \varphi \cdot \nabla w) \mathrm{d} \gamma \mathrm{d} t
\end{aligned}
$$

and

$$
I_{22}=-\int_{0}^{T} \int_{\Omega}\left(s^{2}|\nabla \varphi|^{2}+s \partial_{t} \varphi\right) w(2 s \nabla \varphi \cdot \nabla w) \mathrm{d} x \mathrm{~d} t=\int_{0}^{T} \int_{\Omega} \nabla \cdot\left[\left(s^{3}|\nabla \varphi|^{2}+s^{2} \partial_{t} \varphi\right) \nabla \varphi\right]|w|^{2} \mathrm{~d} x \mathrm{~d} t
$$

Since $\Delta \varphi$ is independent of $x$, by integration by parts in space, we have

$$
I_{13}=-\int_{0}^{T} \int_{\Omega} \Delta w(s \Delta \varphi w) \mathrm{d} x \mathrm{~d} t=s \int_{0}^{T} \int_{\Omega}|\nabla w|^{2} \Delta \varphi \mathrm{d} x \mathrm{~d} t
$$

At last, we have

$$
I_{23}=-\int_{0}^{T} \int_{\Omega}\left(s^{2}|\nabla \varphi|^{2}+s \partial_{t} \varphi\right) w(s \Delta \varphi w) \mathrm{d} x \mathrm{~d} t=-\int_{0}^{T} \int_{\Omega}\left(s^{3}|\nabla \varphi|^{2}+s^{2} \partial_{t} \varphi\right) \Delta \varphi|w|^{2} \mathrm{~d} x \mathrm{~d} t
$$


Gathering all these computations and using the second property in (A.1), we get

$$
\begin{aligned}
& \int_{0}^{T} \int_{\Omega} Q_{+} w Q_{-} w \mathrm{~d} x \mathrm{~d} t=4 s \int_{0}^{T} \int_{\Omega} \theta|\nabla w|^{2} \mathrm{~d} x \mathrm{~d} t \\
& +\int_{0}^{T} \int_{\Omega}\left(\frac{1}{2} \partial_{t}\left(s^{2}|\nabla \varphi|^{2}+s \partial_{t} \varphi\right)+\nabla \cdot\left[\left(s^{3}|\nabla \varphi|^{2}+s^{2} \partial_{t} \varphi\right) \nabla \varphi\right]-\left(s^{3}|\nabla \varphi|^{2}+s^{2} \partial_{t} \varphi\right) \Delta \varphi\right)|w|^{2} \mathrm{~d} x \mathrm{~d} t \\
& +s \int_{0}^{T} \int_{\partial \Omega}|\nabla w|^{2} \nabla \varphi \cdot n \mathrm{~d} \gamma \mathrm{d} t-2 s \int_{0}^{T} \int_{\partial \Omega} \nabla w \cdot n(\nabla \varphi \cdot \nabla w) \mathrm{d} \gamma \mathrm{d} t .
\end{aligned}
$$

For the second term in the right hand side, we notice that the main part in $s^{3}$ is given by

$$
s^{3}\left(\nabla \cdot\left(|\nabla \varphi|^{2} \nabla \varphi\right)-|\nabla \varphi|^{2} \Delta \varphi\right)=s^{3} \nabla\left(|\nabla \varphi|^{2}\right) \cdot \nabla \varphi=8 s^{3} \theta^{3}\left|x-x_{0}\right|^{2} \geq C s^{3} \theta^{3} .
$$

For the boundary terms in (A.7), we notice that, since $z=0$ on $(0, T) \times \partial \Omega, \nabla w=e^{s \varphi} \nabla z$. In particular, $\nabla w \cdot \tau=0$ on $(0, T) \times \partial \Omega$. Thus, we get

$$
s \int_{0}^{T} \int_{\partial \Omega}|\nabla w|^{2} \nabla \varphi \cdot n \mathrm{~d} \gamma \mathrm{d} t-2 s \int_{0}^{T} \int_{\partial \Omega} \nabla w \cdot n(\nabla \varphi \cdot \nabla w) \mathrm{d} \gamma \mathrm{d} t=-s \int_{0}^{T} \int_{\partial \Omega}|\nabla w \cdot n|^{2} \nabla \varphi \cdot n \mathrm{~d} \gamma \mathrm{d} t .
$$

We divide this last integral as follows

$$
-s \int_{0}^{T} \int_{\partial \Omega}|\nabla w \cdot n|^{2} \nabla \varphi \cdot n \mathrm{~d} \gamma \mathrm{d} t=-s \int_{0}^{T} \int_{\Gamma}|\nabla w \cdot n|^{2} \nabla \varphi \cdot n \mathrm{~d} \gamma \mathrm{d} t-s \int_{0}^{T} \int_{\partial \Omega \backslash \Gamma}|\nabla w \cdot n|^{2} \nabla \varphi \cdot n \mathrm{~d} \gamma \mathrm{d} t .
$$

According to (2.5), the second integral is positive and the first integral corresponds to an observation integral. Gathering these estimates, (A.7) becomes, for $s$ large enough

$$
\begin{aligned}
\int_{0}^{T} \int_{\Omega} Q_{+} w Q_{-} w \mathrm{~d} x \mathrm{~d} t \geq & 4 s \int_{0}^{T} \int_{\Omega} \theta|\nabla w|^{2} \mathrm{~d} x \mathrm{~d} t+C s^{3} \int_{0}^{T} \int_{\Omega} \theta^{3}|w|^{2} \mathrm{~d} x \mathrm{~d} t \\
& -s \int_{0}^{T} \int_{\Gamma}|\nabla w \cdot n|^{2} \nabla \varphi \cdot n \mathrm{~d} \gamma \mathrm{d} t .
\end{aligned}
$$

- Step 2 - Bounds on $\Delta w$ and $\partial_{t} w$.

From the definition of $Q_{-}$(A.5), we have

$$
\begin{aligned}
& \frac{1}{2} \int_{0}^{T} \int_{\Omega} \frac{1}{s \theta}\left|\partial_{t} w\right|^{2} \mathrm{~d} x \mathrm{~d} t \leq \int_{0}^{T} \int_{\Omega} \frac{1}{s \theta}\left|Q_{-} w\right|^{2} \mathrm{~d} x \mathrm{~d} t+\int_{0}^{T} \int_{\Omega} \frac{1}{s \theta}|2 s \nabla \varphi \cdot \nabla w+s \Delta \varphi w|^{2} \mathrm{~d} x \mathrm{~d} t \\
& \leq \int_{0}^{T} \int_{\Omega}\left|Q_{-} w\right|^{2} \mathrm{~d} x \mathrm{~d} t+C\left(\int_{0}^{T} \int_{\Omega} s \theta|\nabla w|^{2} \mathrm{~d} x \mathrm{~d} t+\int_{0}^{T} \int_{\Omega} s \theta|w|^{2} \mathrm{~d} x \mathrm{~d} t\right) .
\end{aligned}
$$

In the same way,

$$
\begin{aligned}
& \frac{1}{2} \int_{0}^{T} \int_{\Omega} \frac{1}{s \theta}|\Delta w|^{2} \mathrm{~d} x \mathrm{~d} t \leq \int_{0}^{T} \int_{\Omega} \frac{1}{s \theta}\left|Q_{+} w\right|^{2} \mathrm{~d} x \mathrm{~d} t+\int_{0}^{T} \int_{\Omega} \frac{1}{s \theta}\left(s^{2}|\nabla \varphi|^{2}+s \partial_{t} \varphi\right)^{2}|w|^{2} \mathrm{~d} x \mathrm{~d} t \\
& \leq \int_{0}^{T} \int_{\Omega}\left|Q_{+} w\right|^{2} \mathrm{~d} x \mathrm{~d} t+C \int_{0}^{T} \int_{\Omega} s^{3} \theta^{3}|w|^{2} \mathrm{~d} x \mathrm{~d} t .
\end{aligned}
$$


Thus, coming back to (A.6) and, gathering (A.8) and these last two estimates, we get, for $s$ large enough

$$
\begin{aligned}
\int_{0}^{T} \int_{\Omega} & \left(\frac{1}{s \theta}\left|\partial_{t} w\right|^{2}+\frac{1}{s \theta}|\Delta w|^{2}+s \theta|\nabla w|^{2}+s^{3} \theta^{3}|w|^{2}\right) \mathrm{d} x \mathrm{~d} t \\
& \leq C \int_{0}^{T} \int_{\Omega} e^{2 s \varphi}|f|^{2} \mathrm{~d} x \mathrm{~d} t+C s \int_{0}^{T} \int_{\Gamma}|\nabla w \cdot n|^{2} \nabla \varphi \cdot n \mathrm{~d} \gamma \mathrm{d} t .
\end{aligned}
$$

- Step 3 - Back to the variable $z$.

Since $z=e^{-s \varphi} w$ and according to (A.2), we have, in $\Omega \times(0, T)$

$$
\begin{aligned}
& \left|\partial_{t} z\right|^{2} \leq C e^{-2 s \varphi}\left(\left|\partial_{t} w\right|^{2}+s^{2} \theta^{4}|w|^{2}\right), \quad|\nabla z|^{2} \leq C e^{-2 s \varphi}\left(|\nabla w|^{2}+s^{2} \theta^{2}|w|^{2}\right), \\
& |\Delta z|^{2} \leq C e^{-2 s \varphi}\left(|\Delta w|^{2}+s^{2} \theta^{2}|\nabla w|^{2}+s^{4} \theta^{4}|w|^{2}\right) .
\end{aligned}
$$

Thus, (A.9) gives inequality (2.6) for $s$ large enough.

\section{Appendix B. Proof of the Regularity Result given by Proposition 2.5}

We split the proof in several steps.

- Step 1 - A lifting of the boundary condition of (1.1).

First, we will use a lifting for the boundary condition. Since $g \in H^{1}\left(0, T ; H^{3 / 2}(\partial \Omega)\right) \cap H^{2}\left(0, T ; H^{1 / 2}(\partial \Omega)\right)$, from trace theorem, we deduce that there exists a function $\tilde{u} \in H^{1}\left(0, T ; H^{2}(\Omega)\right) \cap H^{2}\left(0, T ; H^{1}(\Omega)\right)$ such that $\tilde{u}=g$ on $(0, T) \times \partial \Omega$ and

$$
\|\tilde{u}\|_{H^{1}\left(0, T ; H^{2}(\Omega)\right)} \leq C\|g\|_{H^{1}\left(0, T ; H^{3 / 2}(\partial \Omega)\right)}, \quad\|\tilde{u}\|_{H^{2}\left(0, T ; H^{1}(\Omega)\right)} \leq C\|g\|_{H^{2}\left(0, T ; H^{1 / 2}(\partial \Omega)\right)} .
$$

The function $\bar{u}=u-\tilde{u}$ satisfies

$$
\begin{cases}\partial_{t} \bar{u}-\Delta \bar{u}+\bar{u}^{3}+3 \tilde{u} \bar{u}^{2}+3 \tilde{u}^{2} \bar{u}=F, & \text { in }(0, T) \times \Omega, \\ \bar{u}=0, & \text { on }(0, T) \times \partial \Omega, \\ \bar{u}(0, \cdot)=u_{\circ}-\tilde{u}(0, \cdot), & \text { in } \Omega,\end{cases}
$$

with $F$ defined by $F=\sigma h-\partial_{t} \tilde{u}+\Delta \tilde{u}-\tilde{u}^{3}$.

Multiplying the main equation of (B.2) by $\phi \in H_{0}^{1}(\Omega)$ and integrating by parts, we obtain

$$
\begin{aligned}
\int_{\Omega} \partial_{t} \bar{u}(t, x) \phi(x) \mathrm{d} x & +\int_{\Omega} \nabla \bar{u}(t, x) \cdot \nabla \phi(x) \mathrm{d} x+\int_{\Omega} \bar{u}^{3}(t, x) \phi(x) \mathrm{d} x+3 \int_{\Omega} \tilde{u} \bar{u}^{2}(t, x) \phi(x) \mathrm{d} x \\
& +3 \int_{\Omega} \tilde{u}^{2} \bar{u}(t, x) \phi(x) \mathrm{d} x=\int_{\Omega} F(t, x) \phi(x) \mathrm{d} x,
\end{aligned}
$$

a.e. $t \in(0, T)$.

- Step 2 - Finite-dimensional approximated solutions.

At this step, we use the Faedo-Galerkin method and introduce a family of functions $\left\{\phi_{m}\right\}_{m \geq 1}$ in $H_{0}^{1}(\Omega)$ which is an orthogonal basis in $H_{0}^{1}(\Omega)$ and an orthonormal basis in $L^{2}(\Omega)$.

A positive integer $m$ being fixed, we look for an approximated solution of (B.3) $\bar{u}_{m}:[0, T] \rightarrow H_{0}^{1}(\Omega)$ under the form

$$
\bar{u}_{m}(t)=\sum_{i=1}^{m} \alpha_{i m}(t) \phi_{i}
$$


where the coefficients $\left(\alpha_{i m}\right)_{1 \leq i \leq m}$ being to be determined by the conditions:

$$
\begin{aligned}
\int_{\Omega} \partial_{t} \bar{u}_{m}(t, x) \phi_{i}(x) \mathrm{d} x & +\int_{\Omega} \nabla \bar{u}_{m}(t, x) \cdot \nabla \phi_{i}(x) \mathrm{d} x+\int_{\Omega} \bar{u}_{m}^{3}(t, x) \phi_{i}(x) \mathrm{d} x+3 \int_{\Omega} \tilde{u} \bar{u}_{m}^{2}(t, x) \phi_{i}(x) \mathrm{d} x \\
& +3 \int_{\Omega} \tilde{u}^{2} \bar{u}_{m}(t, x) \phi_{i}(x) \mathrm{d} x=\int_{\Omega} F(t, x) \phi_{i}(x) \mathrm{d} x, \quad \forall i=1, \ldots, m
\end{aligned}
$$

along with

$$
\alpha_{i m}(0)=\int_{\Omega} \bar{u}(0, x) \phi_{i}(x) \mathrm{d} x, \quad \forall i=1, \ldots, m
$$

From Picard-Lindelöf theorem (see, for example [26]), the system (B.5)-(B.6) of nonlinear ordinary differential equations, admits a unique local in time solution $\left(\alpha_{i m}\right)_{1 \leq i \leq m}$ in $C^{1}$ defined on a maximal interval $\left(0, T_{m}\right)$.

- Step 3 - A priori estimates.

Multiplying the equation (B.5) by $\alpha_{i m}$, summing over $i$ and integrating on $(0, t)$, we deduce that

$$
\begin{aligned}
\left\|\bar{u}_{m}\right\|_{C^{0}\left(0, t ; L^{2}(\Omega)\right)}+ & \left\|\bar{u}_{m}\right\|_{L^{2}\left(0, t ; H^{1}(\Omega)\right)}+\left\|\bar{u}_{m}\right\|_{L^{4}((0, t) \times \Omega)}^{2} \\
& \leq C\left(\|F\|_{L^{2}((0, T) \times \Omega)}+\|\tilde{u}\|_{L^{4}((0, T) \times \Omega)}^{2}+\|\bar{u}(0, \cdot)\|_{L^{2}(\Omega)}\right) .
\end{aligned}
$$

Thus, the coefficients $\left(\alpha_{i m}\right)_{1 \leq i \leq m}$ stay bounded in $C^{0}\left(0, T_{m}\right)$ and this ensures that they are defined on the global interval $(0, T)$.

- Step 4 - Passage to the limit $m \rightarrow \infty$.

Now, we multiply the equation (B.5) by $\alpha_{i m}^{\prime}$, sum over $i$ and integrate on $(0, T)$. We get that

$$
\begin{aligned}
\left\|\bar{u}_{m}\right\|_{H^{1}\left(0, T ; L^{2}(\Omega)\right)} & +\left\|\bar{u}_{m}\right\|_{L^{\infty}\left(0, T ; H^{1}(\Omega)\right)} \\
& \leq C\left(1+\|\tilde{u}\|_{L^{\infty}((0, T) \times \Omega)}^{2}\right)\left(\|F\|_{L^{2}((0, T) \times \Omega)}+\|\tilde{u}\|_{L^{4}((0, T) \times \Omega)}^{2}+\|\bar{u}(0, \cdot)\|_{H^{1}(\Omega)}\right) .
\end{aligned}
$$

Thus we deduce that, up to a subsequence, $\left(\bar{u}_{m}\right)_{m}$ weakly converges in $H^{1}\left(0, T ; L^{2}(\Omega)\right) \cap L^{\infty}\left(0, T ; H_{0}^{1}(\Omega)\right)$ and strongly converges in $L^{2}((0, T) \times \Omega)$. This convergence properties allow to deduce that the limit $\bar{u}$ satisfies the weak formulation (B.3) and the estimate

$$
\begin{aligned}
\|\bar{u}\|_{H^{1}\left(0, T ; L^{2}(\Omega)\right)}+ & \|\bar{u}\|_{L^{\infty}\left(0, T ; H^{1}(\Omega)\right)}+\|\bar{u}\|_{L^{4}((0, T) \times \Omega)}^{2} \\
& \leq C\left(1+\|\tilde{u}\|_{L^{\infty}((0, T) \times \Omega)}^{2}\right)\left(\|F\|_{L^{2}((0, T) \times \Omega)}+\|\tilde{u}\|_{L^{4}((0, T) \times \Omega)}^{2}+\|\bar{u}(0, \cdot)\|_{H^{1}(\Omega)}\right) .
\end{aligned}
$$

- Step 5 - Higher regularity.

Looking at (B.2) as an elliptic problem, the elliptic regularity implies that $\bar{u}$ belongs to $L^{2}\left(0, T ; H^{2}(\Omega)\right)$ and

$$
\begin{aligned}
\|\bar{u}\|_{L^{2}\left(0, T ; H^{2}(\Omega)\right)} \leq & C\left(1+\|\tilde{u}\|_{L^{\infty}((0, T) \times \Omega)}^{2}\right)\left(\|F\|_{L^{2}((0, T) \times \Omega)}+\|\tilde{u}\|_{L^{4}((0, T) \times \Omega)}^{2}+\|\bar{u}(0, \cdot)\|_{H^{1}(\Omega)}\right) \\
& +C\left(1+\|\tilde{u}\|_{L^{\infty}((0, T) \times \Omega)}^{2}\right)^{3}\left(\|F\|_{L^{2}((0, T) \times \Omega)}+\|\tilde{u}\|_{L^{4}((0, T) \times \Omega)}^{2}+\|\bar{u}(0, \cdot)\|_{H^{1}(\Omega)}\right)^{3} .
\end{aligned}
$$

Moreover, since $\bar{u}$ belongs to $H^{1}\left(0, T ; L^{2}(\Omega)\right) \cap L^{2}\left(0, T ; H^{2}(\Omega)\right)$, according to ([10], Sect. 5.9, Thm. 4), we deduce that $\bar{u}$ belongs to $C^{0}\left(0, T ; H^{1}(\Omega)\right)$.

- Step 6 - Return to the variable $u$. 
Coming back to $u=\bar{u}+\tilde{u}$ and using (B.1), we conclude that

$$
u \in L^{2}\left(0, T ; H^{2}(\Omega)\right) \cap C^{0}\left(0, T ; H^{1}(\Omega)\right) \cap H^{1}\left(0, T ; L^{2}(\Omega)\right)
$$

with the following estimate

$$
\begin{aligned}
\|u\|_{L^{2}\left(0, T ; H^{2}(\Omega)\right)} & +\|u\|_{C^{0}\left(0, T ; H^{1}(\Omega)\right)}+\|u\|_{H^{1}\left(0, T ; L^{2}(\Omega)\right)} \leq C\left(\|\sigma h\|_{L^{2}\left(0, T ; L^{2}(\Omega)\right)}+\|\sigma h\|_{L^{2}\left(0, T ; L^{2}(\Omega)\right)}^{p}\right. \\
& \left.+\|g\|_{H^{1}\left(0, T ; H^{3 / 2}(\partial \Omega)\right)}+\|g\|_{H^{1}\left(0, T ; H^{3 / 2}(\partial \Omega)\right)}^{p}+\left\|u_{\circ}\right\|_{H^{1}(\Omega)}+\left\|u_{\circ}\right\|_{H^{1}(\Omega)}^{p}\right)
\end{aligned}
$$

where the power $p$ is a positive integer that can change from line to line.

- Step 7 - Improved regularity.

Next, let us consider $w=\partial_{t} u$ which is, according to (1.1) and (3.1), formally the solution of

$$
\begin{cases}\partial_{t} w-\Delta w+3 u^{2} w=\sigma \partial_{t} h, & \text { in }(0, T) \times \Omega, \\ w=\partial_{t} g, & \text { on }(0, T) \times \partial \Omega \\ w(0, \cdot)=\Delta u_{\circ}-\left(u_{\circ}\right)^{3}, & \text { in } \Omega\end{cases}
$$

We use the same lifting as in Step 1 and define the function $\bar{w}=w-\partial_{t} \tilde{u}$, which satisfies

$$
\begin{cases}\partial_{t} \bar{w}-\Delta \bar{w}+3 u^{2} \bar{w}=G, & \text { in }(0, T) \times \Omega, \\ \bar{w}=0, & \text { on }(0, T) \times \partial \Omega, \\ \bar{w}(0, \cdot)=w(0, \cdot)-\partial_{t} \tilde{u}(0, \cdot), & \text { in } \Omega,\end{cases}
$$

with $G$ defined by $G=\sigma \partial_{t} h-\partial_{t t} \tilde{u}+\Delta \partial_{t} \tilde{u}-3 u^{2} \partial_{t} \tilde{u}$. For this system, we have a unique solution

$$
\bar{w} \in C^{0}\left(0, T ; L^{2}(\Omega)\right) \cap L^{2}\left(0, T ; H_{0}^{1}(\Omega)\right)
$$

which satisfies

$$
\|\bar{w}\|_{C^{0}\left(0, T ; L^{2}(\Omega)\right)}+\|\bar{w}\|_{L^{2}\left(0, T ; H^{1}(\Omega)\right)} \leq C\left(\|G\|_{L^{2}((0, T) \times \Omega)}+\|\bar{w}(0, \cdot)\|_{L^{2}(\Omega)}\right) .
$$

For the first term in the right hand side, we have

$$
\begin{aligned}
\|G\|_{L^{2}((0, T) \times \Omega)} & \leq\|\sigma h\|_{H^{1}\left(0, T ; L^{2}(\Omega)\right)}+\|\tilde{u}\|_{H^{2}\left(0, T ; L^{2}(\Omega)\right) \cap H^{1}\left(0, T ; H^{2}(\Omega)\right)} \\
& +C\left(\|u\|_{C^{0}\left(0, T ; H^{1}(\Omega)\right)}^{2}+\|\tilde{u}\|_{H^{1}\left(0, T ; H^{2}(\Omega)\right)}\right) .
\end{aligned}
$$

Taking account (B.1) and (B.8), and going back to $w=\bar{w}+\partial_{t} \tilde{u}$, we conclude that

$$
u \in C^{1}\left(0, T ; L^{2}(\Omega)\right) \cap H^{1}\left(0, T ; H^{1}(\Omega)\right),
$$

with the following estimate

$$
\begin{aligned}
& \|u\|_{C^{1}\left(0, T ; L^{2}(\Omega)\right)}+\|u\|_{H^{1}\left(0, T ; H^{1}(\Omega)\right)} \leq C\left(\|\sigma h\|_{H^{1}\left(0, T ; L^{2}(\Omega)\right)}+\|\sigma h\|_{H^{1}\left(0, T ; L^{2}(\Omega)\right)}^{p}\right. \\
& \left.\quad+\|g\|_{H^{1}\left(0, T ; H^{3 / 2}(\partial \Omega)\right) \cap H^{2}\left(0, T ; L^{2}(\partial \Omega)\right)}+\|g\|_{H^{1}\left(0, T ; H^{3 / 2}(\partial \Omega)\right) \cap H^{2}\left(0, T ; L^{2}(\partial \Omega)\right)}^{p}+\left\|u_{\circ}\right\|_{H^{2}(\Omega)}+\left\|u_{\circ}\right\|_{H^{2}(\Omega)}^{p}\right) .
\end{aligned}
$$


Thus, if look at (1.1) as an elliptic problem, we get that $u \in C^{0}\left(0, T ; H^{2}(\Omega)\right)$ and we have the estimate

$$
\begin{aligned}
& \|u\|_{C^{0}\left(0, T ; H^{2}(\Omega)\right)}+\|u\|_{C^{1}\left(0, T ; L^{2}(\Omega)\right)}+\|u\|_{H^{1}\left(0, T ; H^{1}(\Omega)\right)} \\
& \leq C\left(\|\sigma h\|_{H^{1}\left(0, T ; L^{2}(\Omega)\right)}+\|\sigma h\|_{H^{1}\left(0, T ; L^{2}(\Omega)\right)}^{p}+\left\|u_{\circ}\right\|_{H^{2}(\Omega)}+\left\|u_{\circ}\right\|_{H^{2}(\Omega)}^{p}\right. \\
& \left.\quad\|g\|_{H^{1}\left(0, T ; H^{3 / 2}(\partial \Omega)\right) \cap H^{2}\left(0, T ; L^{2}(\partial \Omega)\right)}+\|g\|_{H^{1}\left(0, T ; H^{3 / 2}(\partial \Omega)\right) \cap H^{2}\left(0, T ; L^{2}(\partial \Omega)\right)}^{p}\right) .
\end{aligned}
$$

Let us note that, since $u_{\circ} \in H^{3}(\Omega)$, the initial condition

$$
\bar{w}(0, \cdot)=\Delta u_{\circ}-\left(u_{\circ}\right)^{3}-\partial_{t} \tilde{u}(0, \cdot)
$$

belongs to $H^{1}(\Omega)$. Then, if we multiply the equation (B.10) by $\partial_{t} \bar{w}$ and integrate in $(0, T) \times \Omega$, we obtain that $\partial_{t} \bar{w} \in L^{2}\left(0, T ; L^{2}(\Omega)\right)$ with

$$
\left\|\partial_{t} \bar{w}\right\|_{L^{2}\left(0, T ; L^{2}(\Omega)\right)} \leq C\left(\|G\|_{L^{2}((0, T) \times \Omega)}+\|\bar{w}(0)\|_{H^{1}(\Omega)}\right) .
$$

Hence, if we look at (B.10) as an elliptic problem, we deduce that $\bar{w}$ belongs to $L^{2}\left(0, T ; H^{2}(\Omega)\right)$ with the following estimate

$$
\|\bar{w}\|_{L^{2}\left(0, T ; H^{2}(\Omega)\right)} \leq C\left(\|G\|_{L^{2}((0, T) \times \Omega)}+\|u\|_{C^{0}\left(0, T ; H^{2}(\Omega)\right)}^{2}\|\bar{w}\|_{L^{2}((0, T) \times \Omega)}+\|\bar{w}(0)\|_{H^{1}(\Omega)}\right) .
$$

Besides, since $\bar{w}$ belongs to $H^{1}\left(0, T ; L^{2}(\Omega)\right) \cap L^{2}\left(0, T ; H^{2}(\Omega)\right)$, we deduce that $\bar{w}$ belongs to $C^{0}\left(0, T ; H^{1}(\Omega)\right)$. Coming back to $\partial_{t} u=\bar{w}+\partial_{t} \tilde{u}$, we finally deduce that $u$ belongs to $H^{1}\left(0, T ; H^{2}(\Omega)\right) \cap C^{1}\left(0, T ; H^{1}(\Omega)\right) \cap$ $H^{2}\left(0, T ; L^{2}(\Omega)\right)$ along with the estimate $(2.8)$.

\section{Appendix C. Proof of Lemma 3.8}

On $\widetilde{E}$, we consider the norm $\|\cdot\|_{s}$ defined by

$$
\|z\|_{s}^{2}=\int_{0}^{T} \int_{\Omega} e^{2 s \varphi}\left(\frac{1}{s \theta}\left|\partial_{t} z\right|^{2}+\frac{1}{s \theta}|\Delta z|^{2}+s \theta|\nabla z|^{2}+s^{3} \theta^{3}|z|^{2}\right) \mathrm{d} x \mathrm{~d} t .
$$

For any fixed $C>0$, the set $E_{C}$ is convex and closed in $\left(\widetilde{E},\|\cdot\|_{s}\right)$.

Let $z_{1}$ and $z_{2}$ be given in $E_{C}$. We set $z=z_{1}-z_{2}$. Then, we have

$$
\begin{aligned}
& D J_{0, k}[\mu]\left(z_{1}\right)(z)-D J_{0, k}[\mu]\left(z_{2}\right)(z) \\
= & \int_{0}^{T} \int_{\Omega} e^{2 s \varphi} P_{k} z_{1} D P_{k}\left(z_{1}\right)(z) \mathrm{d} x \mathrm{~d} t+s \int_{0}^{T} \int_{\Gamma} e^{2 s \varphi} \theta\left(\nabla z_{1} \cdot n-\mu\right)(\nabla z \cdot n) \mathrm{d} \gamma \mathrm{d} t \\
& -\int_{0}^{T} \int_{\Omega} e^{2 s \varphi} P_{k} z_{2} D P_{k}\left(z_{2}\right)(z) \mathrm{d} x \mathrm{~d} t-s \int_{0}^{T} \int_{\Gamma} e^{2 s \varphi} \theta\left(\nabla z_{2} \cdot n-\mu\right)(\nabla z \cdot n) \mathrm{d} \gamma \mathrm{d} t \\
= & \int_{0}^{T} \int_{\Omega} e^{2 s \varphi}\left(P_{k} z_{1}-P_{k} z_{2}\right) D P_{k}\left(z_{2}\right)(z) \mathrm{d} x \mathrm{~d} t+\int_{0}^{T} \int_{\Omega} e^{2 s \varphi} P_{k} z_{1}\left(D P_{k}\left(z_{1}\right)(z)-D P_{k}\left(z_{2}\right)(z)\right) \mathrm{d} x \mathrm{~d} t \\
& +s \int_{0}^{T} \int_{\Gamma} e^{2 s \varphi} \theta|\nabla z \cdot n|^{2} \mathrm{~d} \gamma \mathrm{d} t .
\end{aligned}
$$

To estimate the two first terms, we will follow similar computations as in Section 3.4 since we can notice that these terms also appear in (3.37) if we replace $z_{1}, z_{2}$ and $z$ respectively by $w_{k}, Z_{k}$ and $z_{k}$. Since the assumptions 
on the functions are different (in Section 3.4, $w_{k}=\partial_{t} v_{k}$ where $v_{k}$ satisfies (3.4) whereas here $z_{1}$ and $z_{2}$ belong to $E_{C}$ ), we detail the arguments below when they are different from the ones in Section 3.4.

For the first term, we follow the computations made in Step 1 of Section 3.4 and the counterpart of (3.39) is:

$$
\begin{aligned}
& \int_{0}^{T} \int_{\Omega} e^{2 s \varphi}\left(P_{k} z_{1}-P_{k} z_{2}\right) D P_{k}\left(z_{2}\right)(z) \mathrm{d} x \mathrm{~d} t \geq \frac{3}{4} \int_{0}^{T} \int_{\Omega} e^{2 s \varphi}\left|\partial_{t} z-\Delta z\right|^{2} \mathrm{~d} x \mathrm{~d} t \\
& -M \int_{0}^{T} \int_{\Omega} e^{2 s \varphi}|z|^{2} \mathrm{~d} x \mathrm{~d} t-M \int_{0}^{T} \int_{\Omega} e^{2 s \varphi}|\bar{y}|^{2}\left(\left|\partial_{t} u_{k}\right|^{2}+\left|z_{1}\right|^{2}+\left|T_{\bar{M}}^{\prime}\left(y_{2}\right)\right|^{2}\left|\partial_{t} u_{k}\right|^{2}\right) \mathrm{d} x \mathrm{~d} t \\
& -M \int_{0}^{T} \int_{\Omega} e^{2 s \varphi}|\bar{y}|^{2}\left|T_{\bar{M}}^{\prime}\left(y_{2}\right)\right|^{2}\left|z_{2}\right|^{2} \mathrm{~d} x \mathrm{~d} t
\end{aligned}
$$

where

$$
y_{i}(t, x)=v_{k}\left(T_{0}, x\right)+\int_{T_{0}}^{t} z_{i}\left(t^{\prime}, x\right) \mathrm{d} t^{\prime} \text { for } 1 \leq i \leq 2 \text { and } \bar{y}(t, x)=\int_{T_{0}}^{t} z\left(t^{\prime}, x\right) \mathrm{d} t^{\prime}
$$

Using (3.17), we deduce

$$
\begin{aligned}
& \int_{0}^{T} \int_{\Omega} e^{2 s \varphi}\left(P_{k} z_{1}-P_{k} z_{2}\right) D P_{k}\left(z_{2}\right)(z) \mathrm{d} x \mathrm{~d} t \geq \frac{3}{4} \int_{0}^{T} \int_{\Omega} e^{2 s \varphi}\left|\partial_{t} z-\Delta z\right|^{2} \mathrm{~d} x \mathrm{~d} t \\
& -M \int_{0}^{T} \int_{\Omega} e^{2 s \varphi}|z|^{2} \mathrm{~d} x \mathrm{~d} t-M \int_{0}^{T} \int_{\Omega} e^{2 s \varphi}|\bar{y}|^{2}\left(\left|\partial_{t} u_{k}\right|^{2}+\left|z_{1}\right|^{2}+\left|z_{2}\right|^{2}\right) \mathrm{d} x \mathrm{~d} t .
\end{aligned}
$$

For the last term of this inequality, we have

$$
\begin{aligned}
& \int_{0}^{T} \int_{\Omega} e^{2 s \varphi}|\bar{y}|^{2}\left(\left|\partial_{t} u_{k}\right|^{2}+\left|z_{1}\right|^{2}+\left|z_{2}\right|^{2}\right) \mathrm{d} x \mathrm{~d} t \\
& \leq\left\|e^{\varphi} \bar{y}\right\|_{L^{\infty}\left(0, T ; L^{2}(\Omega)\right)}^{2}\left(\left\|\partial_{t} u_{k}\right\|_{L^{2}\left(0, T ; L^{\infty}(\Omega)\right)}^{2}+\left\|z_{1}\right\|_{L^{2}\left(0, T ; L^{\infty}(\Omega)\right)}^{2}+\left\|z_{2}\right\|_{L^{2}\left(0, T ; L^{\infty}(\Omega)\right)}^{2}\right) \\
& \leq M\left\|e^{s \varphi} \bar{y}\right\|_{L^{\infty}\left(0, T ; L^{2}(\Omega)\right)}^{2},
\end{aligned}
$$

according to (3.21) and to the fact that $z_{1}$ and $z_{2}$ belong to $E_{C}$. Using the same arguments than in (3.42) with $z_{k}$ and $\bar{y}_{k}$ replaced respectively by $z$ and $\bar{y}$, we get

$$
\int_{0}^{T} \int_{\Omega} e^{2 s \varphi}|\bar{y}|^{2}\left(\left|\partial_{t} u_{k}\right|^{2}+\left|z_{1}\right|^{2}+\left|z_{2}\right|^{2}\right) \mathrm{d} x \mathrm{~d} t \leq M\left\|e^{s \varphi} z\right\|_{L^{2}\left(0, T ; L^{2}(\Omega)\right)}^{2}
$$

Thus,

$$
\int_{0}^{T} \int_{\Omega} e^{2 s \varphi}\left(P_{k} z_{1}-P_{k} z_{2}\right) D P_{k}\left(z_{2}\right)(z) \mathrm{d} x \mathrm{~d} t \geq \frac{3}{4} \int_{0}^{T} \int_{\Omega} e^{2 s \varphi}\left|\partial_{t} z-\Delta z\right|^{2} \mathrm{~d} x \mathrm{~d} t-M \int_{0}^{T} \int_{\Omega} e^{2 s \varphi}|z|^{2} \mathrm{~d} x \mathrm{~d} t .
$$


For the second term in the right hand side of (C.1), we follow the computations of Step 3 in Section 3.4. We have

$$
\begin{aligned}
& D P_{k}\left(z_{1}\right)(z)-D P_{k}\left(z_{2}\right)(z)=6 z u_{k}\left(T_{\bar{M}}\left(y_{1}\right)-T_{\bar{M}}\left(y_{2}\right)\right)+3 z\left(T_{\bar{M}}\left(y_{1}\right)^{2}-T_{\bar{M}}\left(y_{2}\right)^{2}\right) \\
& +6 \partial_{t} u_{k} u_{k} \bar{y}\left(T_{\bar{M}}^{\prime}\left(y_{1}\right)-T_{\bar{M}}^{\prime}\left(y_{2}\right)\right)+6 \partial_{t} u_{k} \bar{y}\left(T_{\bar{M}}^{\prime}\left(y_{1}\right)-T_{\bar{M}}^{\prime}\left(y_{2}\right)\right) T_{\bar{M}}\left(y_{1}\right) \\
& +6 \partial_{t} u_{k} T_{\bar{M}}^{\prime}\left(y_{2}\right) \bar{y}\left(T_{\bar{M}}\left(y_{1}\right)-T_{\bar{M}}\left(y_{2}\right)\right)+6 u_{k} \bar{y}\left(T_{\bar{M}}^{\prime}\left(y_{1}\right)-T_{\bar{M}}^{\prime}\left(y_{2}\right)\right) z_{1}+6 u_{k} T_{\bar{M}}^{\prime}\left(y_{2}\right) \bar{y} z \\
& +6 \bar{y}\left(T_{\bar{M}}^{\prime}\left(y_{1}\right)-T_{\bar{M}}^{\prime}\left(y_{2}\right)\right) z_{1} T_{\bar{M}}\left(y_{1}\right)+6 T_{\bar{M}}^{\prime}\left(y_{2}\right) \bar{y} z_{1}\left(T_{\bar{M}}\left(y_{1}\right)-T_{\bar{M}}\left(y_{2}\right)\right)+6 T_{\bar{M}}^{\prime}\left(y_{2}\right) \bar{y} z T_{\bar{M}}\left(y_{2}\right) .
\end{aligned}
$$

Using (3.21) and Proposition 3.6 which states the properties satisfied by $T_{\bar{M}}$, we get

$$
\left|D P_{k}\left(z_{1}\right)(z)-D P_{k}\left(z_{2}\right)(z)\right| \leq M\left(|z||\bar{y}|+\left|\partial_{t} u_{k}\right||\bar{y}|^{2}+\left|z_{1}\right||\bar{y}|^{2}\right) .
$$

Thus, for the second term in the right hand side of (C.1), we have the bound

$$
\begin{aligned}
& \int_{0}^{T} \int_{\Omega} e^{2 s \varphi}\left|P_{k} z_{1}\right|\left|D P_{k}\left(z_{1}\right)(z)-D P_{k}\left(z_{2}\right)(z)\right| \mathrm{d} x \mathrm{~d} t \leq M \int_{0}^{T} \int_{\Omega} e^{2 s \varphi}\left|P_{k} z_{1}\right||z||\bar{y}| \mathrm{d} x \mathrm{~d} t \\
& +M \int_{0}^{T} \int_{\Omega} e^{2 s \varphi}\left|P_{k} z_{1}\right|\left(\left|\partial_{t} u_{k}\right|+\left|z_{1}\right|\right)|\bar{y}|^{2} \mathrm{~d} x \mathrm{~d} t .
\end{aligned}
$$

For the first term in the right hand side of (C.3), we have

$$
\begin{aligned}
\int_{0}^{T} \int_{\Omega} e^{2 s \varphi}\left|P_{k} z_{1}\|z\| \bar{y}\right| \mathrm{d} x \mathrm{~d} t & \leq C\left\|P_{k} z_{1}\right\|_{L^{2}((0, T) \times \Omega)}\left\|e^{2 s \varphi} z \bar{y}\right\|_{L^{2}((0, T) \times \Omega)} \\
& \leq C\left\|P_{k} z_{1}\right\|_{L^{2}((0, T) \times \Omega)}\left\|e^{s \varphi} z\right\|_{L^{2}\left(0, T ; L^{6}(\Omega)\right)}\left\|e^{s \varphi} \bar{y}\right\|_{L^{\infty}\left(0, T ; L^{3}(\Omega)\right)} \\
& \leq C\left\|P_{k} z_{1}\right\|_{L^{2}((0, T) \times \Omega)}\left(\left\|e^{s \varphi} z\right\|_{L^{2}\left(0, T ; L^{6}(\Omega)\right)}^{2}+\left\|e^{s \varphi} \bar{y}\right\|_{L^{\infty}\left(0, T ; L^{3}(\Omega)\right)}^{2}\right) \\
& \leq M\left(\left\|e^{s \varphi} z\right\|_{L^{2}\left(0, T ; L^{6}(\Omega)\right)}^{2}+\left\|e^{s \varphi} \bar{y}\right\|_{L^{\infty}\left(0, T ; L^{3}(\Omega)\right)}^{2}\right)
\end{aligned}
$$

where we have used that $z_{1}$ belongs to $E_{C}$. For the second term in the right hand side of (C.3), we have

$$
\begin{aligned}
& \int_{0}^{T} \int_{\Omega} e^{2 s \varphi}\left|P_{k} z_{1}\right|\left(\left|\partial_{t} u_{k}\right|+\left|z_{1}\right|\right)|\bar{y}|^{2} \mathrm{~d} x \mathrm{~d} t \leq C\left\|P_{k} z_{1}\right\|_{L^{2}((0, T) \times \Omega)}\left\|\left(\left|\partial_{t} u_{k}\right|+\left|z_{1}\right|\right) e^{2 s \varphi}|\bar{y}|^{2}\right\|_{L^{2}((0, T) \times \Omega)} \\
& \leq C\left\|P_{k} z_{1}\right\|_{L^{2}((0, T) \times \Omega)}\left(\left\|\partial_{t} u_{k}\right\|_{L^{2}\left(0, T ; L^{\infty}(\Omega)\right)}+\left\|z_{1}\right\|_{L^{2}\left(0, T ; L^{\infty}(\Omega)\right)}\right)\left\|e^{2 s \varphi}|\bar{y}|^{2}\right\|_{L^{\infty}\left(0, T ; L^{2}(\Omega)\right)} \\
& \leq M\left\|e^{s \varphi} \bar{y}\right\|_{L^{\infty}\left(0, T ; L^{4}(\Omega)\right)}^{2},
\end{aligned}
$$

where we have used that $z_{1}$ belongs to $E_{C}$ and $u_{k}$ satisfies (3.21). Therefore, inequality (C.3) becomes

$$
\int_{0}^{T} \int_{\Omega} e^{2 s \varphi}\left|P_{k} z_{1} \| D P_{k}\left(z_{1}\right)(z)-D P_{k}\left(z_{2}\right)(z)\right| \mathrm{d} x \mathrm{~d} t \leq M\left(\left\|e^{s \varphi} z\right\|_{L^{2}\left(0, T ; L^{6}(\Omega)\right)}^{2}+\left\|e^{s \varphi} \bar{y}\right\|_{L^{\infty}\left(0, T ; L^{4}(\Omega)\right)}^{2}\right) .
$$

For the terms in the right hand side, we first have

$$
\left\|e^{s \varphi} z\right\|_{L^{2}\left(0, T ; L^{6}(\Omega)\right)}^{2} \leq\left\|e^{s \varphi} z\right\|_{L^{2}\left(0, T ; H^{1}(\Omega)\right)}^{2} \leq \int_{0}^{T} \int_{\Omega} e^{2 s \varphi}\left(\left(1+s^{2} \theta^{2}\right)|z|^{2}+|\nabla z|^{2}\right) \mathrm{d} x \mathrm{~d} t
$$


thanks to (A.2). Moreover,

$$
\left\|e^{s \varphi} \bar{y}\right\|_{L^{\infty}\left(0, T ; L^{4}(\Omega)\right)}^{2} \leq C\left\|e^{s \varphi} \bar{y}\right\|_{L^{\infty}\left(0, T ; H^{1}(\Omega)\right)}^{2} \leq C \sup _{0 \leq t \leq T} \int_{\Omega} e^{2 s \varphi}\left(\left(1+s^{2} \theta^{2}\right)|\bar{y}|^{2}+|\nabla \bar{y}|^{2}\right) \mathrm{d} x .
$$

We notice that, for all $t \in(0, T)$

$$
\begin{aligned}
& \int_{\Omega} e^{2 s \varphi(t, x)}\left(\left(1+s^{2} \theta^{2}(t, x)\right)|\bar{y}(t, x)|^{2}+|\nabla \bar{y}(t, x)|^{2}\right) \mathrm{d} x \\
& \leq\left. C \int_{\Omega} e^{2 s \varphi(t, x)}\left(1+s^{2} \theta^{2}(t, x)\right)\left|\int_{T_{0}}^{t}\right| z\left(t^{\prime}, x\right)\right|^{2} \mathrm{~d} t^{\prime}\left|\mathrm{d} x+C \int_{\Omega} e^{2 s \varphi(t, x)}\right| \int_{T_{0}}^{t}\left|\nabla z\left(t^{\prime}, x\right)\right|^{2} \mathrm{~d} t^{\prime} \mid \mathrm{d} x \\
& \leq C \int_{0}^{T} \int_{\Omega} e^{2 s \varphi(t, x)}\left(1+s^{2} \theta^{2}(t, x)\right)|z(t, x)|^{2} \mathrm{~d} x \mathrm{~d} t+C \int_{0}^{T} \int_{\Omega} e^{2 s \varphi(t, x)}\left|\nabla z\left(t^{\prime}, x\right)\right|^{2} \mathrm{~d} x \mathrm{~d} t
\end{aligned}
$$

according to the fact that, in both cases (2.1) and (2.4), for $s$ large enough, for all $t^{\prime}$ between $T_{0}$ and $t$, for all $x \in \Omega$

$$
e^{2 s \varphi(t, x)}\left(1+s^{2} \theta^{2}(t, x)\right) \leq e^{2 s \varphi\left(t^{\prime}, x\right)}\left(1+s^{2} \theta^{2}\left(t^{\prime}, x\right)\right) .
$$

Thus, inequality (C.4) becomes

$$
\int_{0}^{T} \int_{\Omega} e^{2 s \varphi}\left|P_{k} z_{1}\right|\left|D P_{k}\left(z_{1}\right)(z)-D P_{k}\left(z_{2}\right)(z)\right| \mathrm{d} x \mathrm{~d} t \leq M \int_{0}^{T} \int_{\Omega} e^{2 s \varphi}\left(\left(1+s^{2} \theta^{2}\right)|z|^{2}+|\nabla z|^{2}\right) \mathrm{d} x \mathrm{~d} t .
$$

Coming back to (C.1), using (C.2), this last inequality and the Carleman estimate (2.1), we conclude that, for $s$ large enough, there exists $\delta>0$ such that, for all $z_{1}$ and $z_{2}$ in $E_{C}$

$$
D J_{0, k}[\mu]\left(z_{1}\right)\left(z_{1}-z_{2}\right)-D J_{0, k}[\mu]\left(z_{2}\right)\left(z_{1}-z_{2}\right) \geq \delta\left\|z_{1}-z_{2}\right\|_{s}^{2} .
$$

This proves that $J_{0, k}[\mu]$ is strongly convex in $E_{C}$.

\section{REFERENCES}

[1] L. Baudouin, M. de Buhan and S. Ervedoza, Global Carleman estimates for waves and applications. Commun. Partial Differ. Equ. 38 (2013) 823-859.

[2] L. Baudouin, M. de Buhan and S. Ervedoza, Convergent algorithm based on Carleman estimates for the recovery of a potential in the wave equation. SIAM J. Numer. Anal. 55 (2017) 1578-1613.

[3] M. Boulakia, C. Grandmont and A. Osses, Some inverse stability results for the bistable reaction-diffusion equation using Carleman inequalities. C. R. Math. Acad. Sci. Paris 347 (2009) 619-622.

[4] P.N. Brown and V. Saad, Convergence theory of non linear Newton-Krylov algorithms. SIAM J. Optim. 4 (1994) $297-330$.

[5] A.L. Bukhgelm and M.V. Klibanov, Uniqueness in the large of a class of multidimensional inverse problems. Dokl. Akad. Nauk SSSR 260 (1981) 269-272.

[6] S. Butterworth, On the theory of filter amplifiers. Wirel. Eng. 7 (1930) 536-541.

[7] N. Cîndea, E. Fernández-Cara and A. Münch, Numerical controllability of the wave equation through primal methods and Carleman estimates. ESAIM: COCV 19 (2013) 1076-1108.

[8] P. Colli Franzone, L.F. Pavarino and S. Scacchi, Mathematical cardiac electrophysiology. Vol. 13 of MSEA. Modeling, Simulation and Applications. Springer, Cham (2014).

[9] H. Egger, H.W. Engl and M.V. Klibanov, Global uniqueness and Hölder stability for recovering a nonlinear source term in a parabolic equation. Inverse Probl. 21 (2005) 271-290.

[10] L.C. Evans, Partial differential equations. Vol. 19 of Graduate Studies in Mathematics. American Mathematical Society, Providence, RI, second edition (2010).

[11] E. Fernández-Cara, M. González-Burgos, S. Guerrero and J.-P. Puel, Exact controllability to the trajectories of the heat equation with Fourier boundary conditions: the semilinear case. ESAIM: COCV 12 (2006) 466-483. 
[12] E. Fernández-Cara, A. Münch and D.A. Souza, On the numerical controllability of the two-dimensional heat, Stokes and Navier-Stokes equations. J. Sci. Comput. 70 (2017) 819-858.

[13] A.V. Fursikov and O.Yu. Imanuvilov, Controllability of evolution equations. Vol. 34 of Lecture Notes Series. Seoul National University, Research Institute of Mathematics, Global Analysis Research Center, Seoul (1996).

[14] L.F. Ho, Observabilité frontière de l'équation des ondes. C. R. Acad. Sci. Paris Sér. I Math. 302 (1986) $443-446$.

[15] O.Y. Imanuvilov and M. Yamamoto, Lipschitz stability in inverse parabolic problems by the Carleman estimate. Inverse Prob. 14 (1998) 1229-1245.

[16] K. Ito and B. Jin, Inverse problems. Vol. 22 of Series on Applied Mathematics. World Scientific Publishing Co. Pte. Ltd., Hackensack, NJ (2015). Tikhonov theory and algorithms.

[17] M.V. Klibanov, Global convexity in a three-dimensional inverse acoustic problem. SIAM J. Math. Anal. 28 (1997) 1371-1388.

[18] M.V. Klibanov, Carleman estimates for global uniqueness, stability and numerical methods for coefficient inverse problems. $J$. Inverse Ill-Posed Probl. 21 (2013) 477-560.

[19] M.V. Klibanov and V.G. Kamburg, Globally strictly convex cost functional for an inverse parabolic problem. Math. Methods Appl. Sci. 39 (2016) 930-940.

[20] M.V. Klibanov, A.E. Kolesov and D.-L. Nguyen, Convexification method for an inverse scattering problem and its performance for experimental backscatter data for buried targets. SIAM J. Imag. Sci. 12 (2019) 576-603.

[21] D.A. Knoll and D.E. Keyes, Jacobian-free Newton-Krylov methods: a survey of approaches and applications. J. Comput. Phys. 193 (2004) 357.

[22] T.T. Le and L.H. Nguyen, A convergent numerical method to recover the initial condition of nonlinear parabolic equations from lateral Cauchy data. preprint.

[23] J.-L. Lions, Contrôlabilité exacte, perturbations et stabilisation de systèmes distribués. Tome 1. Contrôlabilité exacte. [Exact controllability], With appendices by E. Zuazua, C. Bardos, G. Lebeau and J. Rauch. Vol. 8 of Recherches en Mathématiques Appliquées [Research in Applied Mathematics]. Masson, Paris (1988).

[24] J.L. Lions and E. Magenes, Non-homogeneous boundary value problems and applications. In Vol. 1. Springer Science \& Business Media (2012).

[25] A. Savitzky and M. Golay, Smoothing and differentiation of data by simplified least squares procedures. Anal. Chem. 8 (1964) $1627-1639$.

[26] K. Schmitt and R. Thompson, Nonlinear analysis and differential equations: An introduction. Lecture Notes. University of Utah, Department of Mathematics (1998).

[27] M. Yamamoto, Carleman estimates for parabolic equations and applications. Inverse Problems 25 (2009) 123013. 